\title{
Kondo Effect in Flux Phases
}

\author{
Carlos R. Cassanello and Eduardo Fradkin \\ Loomis Laboratory of Physics and Materials Research Laboratory \\ University of Illinois at Urbana-Champaign \\ 1110 W.Green St., Urbana, IL, 61801-3080
}

\begin{abstract}
We consider a band of fermions in two space dimensions with a flux phase (relativistic) dispersion relation coupled to a local magnetic impurity via an $s-d$ interaction. This model describes spinons of a flux phase and it is also a qualitative model of the quasiparticles in a $d_{x^{2}-y^{2}}$ superconductor. We find a zero-temperature phase transition at a finite coupling constant between a weak coupling unscreened impurity state and a strong coupling regime with a Kondo effect. We use large- $N$ methods to study the phase transition in this Kondo system away from marginality. The Kondo energy scales linearly with the distance to the transition . The zero-field magnetic suceptibility at zero temperature diverges linearly. Similar behavior is found in the $T$ matrix which shows a resonance at the Kondo scale. However, in addition to this simple scaling, we always find the presence of logarithmic corrections-toscaling. Such behavior is typical of systems at an upper critical dimension. We derive an effective fermion model in one space dimension for this problem. Unlike the usual Kondo problem, this system has an intrinsic multichannel nature which follows from the spinor structure of 2+1-dimensional relativistic fermions.
\end{abstract}

PACS numbers: 71.27.+a,75.20.Hr

Typeset using REVTEX 


\section{INTRODUCTION}

It is by now well understood that the presence of a small concentration of magnetic impurities into an otherwise non-magnetic metallic host can affect dramatically the lowtemperature properties of the system. The prototype of these interesting phenomena is the Kondo Effect. [1] At the single-impurity level, there is a non-perturbative crossover between a Curie-Weiss law behavior (in which the impurity behaves like a non-interacting localized magnetic moment) at high temperature, and a strongly interacting regime in which the magnetic impurity and the band electrons form a singlet ground state. In other words, in this low-temperature (strong-coupling) regime, the band electrons conspire to screen out the spin (and magnetic moment) of the impurity. At zero temperature a similar crossover occurs as a function of an external magnetic field. This picture has been developed by the concerted use of the renormalization group [2, [3], "exact" numerical simulations and scaling [4], exact solutions via the Bethe-Ansatz [5, 6] and large- $N$ expansions [7,8].

From the point of view of scaling, the Kondo problem is a typical situation in which a trivial fixed point, which describes band electrons decoupled from the magnetic impurity, is destabilized by a marginally relevant perturbation, the coupling to the magnetic impurity. This leads to an asymptotically free renormalization group flow with a $\beta$-function which is quadratic in the coupling constant. Marginal perturbations appear in critical systems at a critical dimension. The standard $s-d$ Kondo Hamiltonian is effectively a model of one dimensional chiral fermions coupled to a single magnetic impurity through the forward scattering channel [5]. Clearly, in this case we are at the lowest critical dimension. This is a direct consequence of the fact that the band electrons have a Fermi surface where the density of states is finite and essentially constant. Thus, the Kondo effect is ultimately due to the availability of states in the electron band which can efficiently screen the impurity spin no matter how weak the exchange coupling constant may be.

Some time ago Withoff and Fradkin (WF) [9] considered a genaralization of the Kondo problem to systems in which the density of band electron states may actually go to zero at 
the Fermi energy. They showed that if the density of states of the electron band vanishes at the Fermi energy as a positive power of the energy, the Kondo effect is suppressed for small values of the exchange constant and that the Kondo screening only happens beyond a critical value of this coupling. In fact, it is easy to see that the exponent $r$ of the one particle density of states, $N(E) \propto\left|E-E_{F}\right|^{r}$ plays a role here quite analogous to the distance to the lower critical dimension $d-d_{c}$ in critical phenomena. WF showed, using a combination of a "poor man's scaling argument" and a large- $N$ limit, that at least for small values of the exponent $r$, this is the correct picture. Quite generally, if the exponent $r>0$ this is a non-marginal Kondo system.

There are a number of systems of physical interest where this situation does arise. A simple example are the fermionic excitations of a quantum antiferromagnet in a flux phase [10]. More importantly, the normal state excitations of a $d$-wave superconductor (with symmetry $\left.d_{x^{2}-y^{2}}\right)$ behave precisely in this fashion [11]. In the vicinity of each node of the gap function (hence, the use of the term "node" hereafter), the dispersion relation for the normal quasiparticles is linear in the momentum. Thus, sufficiently close to the node, the quasiparticles have an effective relativistic-like dispersion. In the theory of superconductivity (isotropic or not) [12] the dynamics of the quasiparticles is usually pictured in terms of Nambu spinors. For the case of a d-wave superconductor, Nersesyan, Tsvelik and Wenger [13 have shown that this approach leads to effective Hamiltonian for the quasiparticles which takes the form of a massless Dirac Hamiltonian for each node of the gap, with the "speed of light" equal to the Fermi velocity. (Naturally, relativistic massless Dirac fermions themselves always have this property.) In this paper we consider a model which describes properly the coupling of flux-phase fermions to a magnetic impurity. It turns out that this model can also be used to describe the coupling of a local magnetic impurity to a d-wave superconductor, including pair-breaking effects. In a separate publication we will discuss in detail the problem of a magnetic impurity in a $d$-wave superconductor in more detail [14,15].

In this paper we reconsider the Kondo effect for non-marginal systems. The model has fermions coupled locally to a magnetic impurity. The fermions are assumed to obey 
a relativistic-like dispersion law and hence a density of states vanishing linearly with the energy. For simplicity we consider models with just one species of "relativistic" fermions. We will refer to them as "having a single node". In particular we will discuss the case of an impurity coupled to an electron band with a density of states that vanishes linearly with the distance to Fermi energy $E_{F} \equiv 0$. This case was not examined by WF who found that the singularity structure changed as soon as $r>1 / 2$. We will show in this paper that at $r=1$ the additional singularities conspire to give simple scaling laws modified by logarithmic corrections [16]. This picture is strongly reminiscent of a critical system at an upper critical dimension.

As in the conventional Kondo problem, here too we can construct an effective onedimensional theory. However, when one carefully reduces the $2+1$-dimensional fermions with a relativistic-like energy-momentum dispersion to an effective $1+1$-dimensional model, one finds that there are at least two angular momentum channels that are always coupled. Thus, these are all inherently multichannel Kondo systems. In particular, it is always the $\ell=0$ channel coupled either to $\ell=1$ channel or to $\ell=-1$ channel. Which pair of angular momentum channels are actually coupled depends on the Parity of the node (or cone) to which the channels belong. We find two equivalent ways to represent the dimensionally reduced model. We have the freedom to choose the effective $1+1$-dimensional fermions to have a local kinetic energy and, hence, to behave like conventional right movers. However, in this picture, the effective interaction with the impurity becomes non-local. The other, alternative picture, is to have a local coupling between right moving fermions which now have a non-local kinetic energy. In fact, due to phase space factors, the density of states of the effective $1+1$-dimensional model goes to zero linearly with energy. The linearity of the density of states reflects the fact that in the original problem the fermions move in two-space dimensions. It is this feature what drives the system out of marginality and what generates a critical coupling constant below which the width of the Kondo resonance vanishes.

In all cases of physical interest, Parity is an exact symmetry. This means that the number of nodes (cones) is even and that there should be as many cones with positive 
parity as there are with negative parity. In the case of both the $d$-wave superconductors and of the flux-phase fermions, this property follows from the alternating signs of the gap function. In contrast, if either parity or time reversal were broken, all four cones would have the same properties under parity. However, in that case there would always be a non-zero energy gap on the entire Fermi surface. Thus, in general, the effective Kondo hamiltonians always have an exact degeneracy (angular momentum channels). The spin symmetry is the usual $S U(2)$ spin rotation invariance (which here we call color). Here we will work with the $S U\left(N_{c}\right)$ generalization of this symmetry, with the physical $N_{c}=2$. The angular momentum degeneracy leads to and $S U\left(N_{f}\right)$ flavor degeneracy. For a problem with one node, $N_{f}=2$. When more than one node is considered, $N_{f}>2$. We will also consider impurity scattering amplitudes which may change the angular momentum of the fermions. The basic and simplest model is worked out in section [II].

In the second part of the paper we use large- $N$ methods to investigate the behavior of these systems near the critical coupling [7]. We find that there is still a Kondo scale once we go over the critical coupling. However, the position and the width of the resonance are not related anymore in the very simple way they are in the usual Kondo effect. The details are worked out in section [II. The most salient feature of our results is the presence of logarithmic corrections to simple scaling in all quantities of physical interest, including the zero-field paramagnetic suceptibility (at $T=0$ ), the Kondo scale and the $T$-matrix for bulk fermions.

The large- $N_{c}$ theory that we present here suggests the following scenario. For small values of the coupling constant $J$, the impurity is free and effectively paramagnetic. In contrast, for large values of $J$, the impurity is screened by the fermions. The critical coupling $J_{c}$ is dimensionful and scales with the energy cutoff. However, the fact that these systems are inherently multichannel Kondo systems suggests that for $J \geq J_{c}$ the impurity is actually overscreened [17]. In the language of the Renormalization Group this requires a flow with two finite fixed points: one infrared unstable fixed point for the paramagnetic-Kondo phase transition and an infrared stable to describe the multichannel behavior of the Kondo phase. 
As in the conventional Kondo problem, the large- $N_{c}$ theory can only describe the formation of the Kondo singlet which in a non-marginal system can only take place at finite coupling. In marginal Kondo systems multichannel behavior is found as a next-to-leading order effect in the $1 / N_{c}$ expansion or to third order in perturbative scaling [18]. In the systems of

physical interest mentioned above where this model applies, the number of channels $N_{f}$ is always larger than the number of colors. In such a situation one can imagine that there exists a critical number of channels such that both fixed points actually coincide and below this critical number the transition dissapears altogether [19]. We should emphasize here that this physics cannot be accessed by a straightforward use of the large- $N_{c}$ expansion.

The paper is organized as follows. In section II we derive the effective $1+1$-dimensional impurity models for systems of fermions in two space dimensions with relativistic-like dispersion (flux phase). In section III we study these models in the large- $N$ limit and determine their critical behavior. In section $\mathbb{\square}$ we calculate the propagator for the band fermions in the $N \rightarrow \infty$ limit and use it to derive the $T$-matrix. In section $\nabla$ we consider a model with the most general type of scattering process for fermions with only one node coupled to a single impurity and determine the phase diagram. Section $\nabla]$ is devoted to the conclusions and to the discussions of the similarities and differences between the problem we discuss here and the conventional Kondo problem. In Appendix A we sketch the calculations of a few integrals.

\section{TOY MODEL: S-D HAMILTONIAN FOR A LINEAR DENSITY OF STATES}

In this section we want to study the s-d Hamiltonian a la Read and Newns [7], with the difference that we are not assuming a constant density of states for the conduction fermions. We are coupling an f-impurity to a bath of "relativistic" electrons, ie., electrons with a linear dispersion relation. We assume the chemical potential for the electrons (the Fermi energy) is zero. This is a very special case but it will turn out to be quite important for the case of a d-wave superconductor as it will be discussed in the following sections. 
We start with a two-dimensional free fermion Hamiltonian with a linear spectrum and a Fermi velocity $v_{F}$ which may correspond to the linearization of a fermion band of width $2 D$, where $D$ is an energy cutoff. We will consider a model of band fermions with $N_{c}=N$ spin components with $N=2$ for the physically relevant case of spin $S U(2)$. In general, this model will have a global $S U\left(N_{c}\right)$ "color" (or spin) symmetry. This model has a well defined large- $N$ limit. The free part of the Hamiltonian is

$$
H_{0}=\int \frac{d^{2} p}{(2 \pi)^{2}} \Psi_{\sigma i}^{\dagger}(p)\left(v_{F} \vec{p} \cdot \vec{\alpha}\right)_{i j} \Psi_{\sigma j}(p)
$$

where $\alpha_{1}$ and $\alpha_{2}$ are Pauli matrices, so that

$$
\vec{p} \cdot \vec{\alpha}=p_{1} \alpha_{1}+p_{2} \alpha_{2}=p\left(\begin{array}{cc}
0 & e^{-i \theta} \\
e^{i \theta} & 0
\end{array}\right)
$$

Eq.(2.2) can be diagonalized by expanding the fields into a linear combination of spinor eigenstates. Let's call them $u_{ \pm}$. This eigenmodes satisfy

$$
v_{F} \vec{p} \cdot \vec{\alpha} u_{ \pm}= \pm v_{F}|p| u_{ \pm}
$$

A particular (and convenient) choice of eigenstates is given by

$$
u_{ \pm}^{(1)}=\frac{1}{\sqrt{2}} \quad \text { and } \quad u_{ \pm}^{(2)}= \pm \frac{e^{i \theta}}{\sqrt{2}}
$$

The fields $\Psi$ 's carry a spin index $\sigma$ apart from the spinor index $i$ and $j$ which we ignore at this point but we shall put it back in when dealing with the interaction with the impurity term.

$$
\Psi_{i}(p)=\sum_{\lambda= \pm} u_{i}^{\lambda}(p) \zeta_{\lambda}(p)
$$

so that

$$
\begin{aligned}
& \Psi_{1}(p)=\frac{1}{\sqrt{2}}\left[\zeta_{+}(p)+\zeta_{-}(p)\right] \\
& \Psi_{2}(p)=\frac{1}{\sqrt{2}}\left[\zeta_{+}(p)-\zeta_{-}(p)\right] e^{i \theta}
\end{aligned}
$$

Now we can expand the $\zeta$ fields in a basis of angular momentum eigenmodes 


$$
\zeta_{ \pm}(p)=\sum_{m=-\infty}^{\infty} e^{i m \theta} \zeta_{ \pm, m}(p)
$$

where $m$ is an integer and \pm indicates the positive/negative energy spinor eigenmodes of the Dirac equation given by Eq.(2.1). We then have

$$
H_{0}=\sum_{m=-\infty}^{\infty} \int_{0}^{\infty} \frac{p d p}{2 \pi} v_{F}|p|\left[\zeta_{+, m}^{\dagger}(p) \zeta_{+, m}(p)-\zeta_{-, m}^{\dagger}(p) \zeta_{-, m}(p)\right]
$$

In real space we have

$$
\Psi_{i}(\vec{r})=\int_{0}^{\infty} \frac{p d p}{2 \pi} \sum_{\lambda= \pm} \int_{0}^{2 \pi} \frac{d \theta}{2 \pi} \sum_{m=-\infty}^{\infty} u_{i}^{\lambda}(\theta) e^{i \vec{p} \cdot \vec{r}} e^{i m \theta} \zeta_{m, \lambda}(p)
$$

To reduce to an equivalent one-dimensional problem we use polar coordinates where $\vec{p} \equiv$ $(p, \theta), \vec{r} \equiv(r, \phi)$ and $\vec{p} \cdot \vec{r}=p r \cos (\theta-\phi)$. By making use of the expansion

$$
e^{i z \cos \varphi}=\sum_{n=-\infty}^{\infty} i^{n} J_{n}(z) e^{i n \varphi}
$$

where $J_{n}(z)$ are the Bessel functions. They satisfy the relations

$$
\int_{0}^{2 \pi} \frac{d \theta}{2 \pi} e^{i p r \cos (\theta-\phi)+i m \theta}=i^{m} J_{m}(p r) e^{i m \phi} \quad \text { since } \quad J_{-n}(z)=(-1)^{n} J_{n}(z)
$$

Using these in Eq.(2.9) one gets

$$
\begin{aligned}
& \Psi_{1}(r, \phi)=\int_{0}^{\infty} \frac{p d p}{2 \pi} \frac{1}{\sqrt{2}} \sum_{m=-\infty}^{\infty} i^{m} J_{m}(p r) e^{-i m \phi}\left[\zeta_{m,+}(p)+\zeta_{m,-}(p)\right] \\
& \Psi_{2}(r, \phi)=\int_{0}^{\infty} \frac{p d p}{2 \pi} \frac{1}{\sqrt{2}} \sum_{m=-\infty}^{\infty} i^{m+1} J_{m+1}(p r) e^{-i(m+1) \phi}\left[\zeta_{m,+}(p)-\zeta_{m,-}(p)\right]
\end{aligned}
$$

At the impurity site, i.e., for $r \rightarrow 0, J_{0}(0)=1$ and $J_{m}(0)=0$ for $m \neq 0$. So, we are left with

$$
\begin{aligned}
& \Psi_{1}(0)=\int_{0}^{\infty} \frac{p d p}{2 \pi} \frac{1}{\sqrt{2}}\left[\zeta_{0,+}(p)+\zeta_{0,-}(p)\right] \\
& \Psi_{2}(0)=\int_{0}^{\infty} \frac{p d p}{2 \pi} \frac{1}{\sqrt{2}}\left[\zeta_{-1,+}(p)-\zeta_{-1,-}(p)\right]
\end{aligned}
$$

This is telling us that the effect of having a cone-like dispersion relation for the fermions instead of a flat band will not only reduce the available density of states but it will also induce an angular momentum mode mixing. Even though we may regard the impurity as having a 
$\delta$-function spatial form factor as in the case of the usual Kondo effect, the coupling to the fermions will no longer be restricted only to the s-wave, or the spherically symmetric angular momentum channel. This feature will become evident in the following step. We want to rewrite the theory by introducing effective one-dimensional fermion operators. We use the radial component of incoming and outgoing waves. We find two "flavors" of right-movers given by

$$
\begin{array}{ll}
c_{1}(p) \equiv \sqrt{|p|} \zeta_{+, 0}(|p|) ; & c_{2}(p) \equiv \sqrt{|p|} \zeta_{+,-1}(|p|) ; \quad \text { for } p>0 \\
c_{1}(p) \equiv \sqrt{|p|} \zeta_{-, 0}(|p|) ; & c_{2}(p) \equiv-\sqrt{|p|} \zeta_{-,-1}(|p|) ; \text { for } p<0
\end{array}
$$

In other words, $c_{1}$ are the fermion states with angular momentum $\ell=0$ while $c_{2}$ are the fermion states with angular momentum $\ell=-1$. We summarize these mappings as

$$
\Psi_{i}(0)=\frac{1}{\sqrt{2}} \int_{-\infty}^{\infty} \frac{d p}{2 \pi} \sqrt{|p|} c_{i}(p)
$$

For a system with an $S U\left(N_{c}\right)$ symmetry, the s-d interaction can be written in the form

$$
H_{\mathrm{imp}}=\Psi_{\alpha}^{\dagger}(0) \tau_{\alpha \beta}^{\mathrm{a}} \Psi_{\beta}(0) S^{a} \equiv \Psi_{\alpha}^{\dagger}(0) \tau_{\alpha \beta}^{a} \Psi_{\beta}(0) f_{\gamma}^{\dagger} \tau_{\gamma \delta}^{a} f_{\delta}
$$

where $f_{\gamma}^{\dagger} \tau_{\gamma \delta}^{a} f_{\delta}$ is the impurity spin coupled to the electrons at the real-space position $x=0$. For a system with an $S U\left(N_{c}\right)$ symmetry, $a=1, \ldots, N_{c}^{2}-1$. We will use the standard representation of spin operators (here, the generators of $S U\left(N_{c}\right)$ defined in terms of the fermion operators $f_{\gamma}$ as $f_{\gamma}^{\dagger} \tau_{\gamma \delta}^{a} f_{\delta}$. If the constraint $f_{\gamma}^{\dagger} f_{\gamma}=1$ is satisfied, the impurity is in the lowest (fundamental) representation of $S U\left(N_{c}\right)$. Other representations can be constructed by changing the "filling fraction" of the impurity spin. Notice that, for $S U(2)$, the only possible value of the filling fraction is equal to one.

Once again we stress the fact that the angular momentum channels given by $m=0$ and $m=-1$ couple to the impurity. This is an important difference with respect to the case of constant density of states, where only the $m=0$ channel couples to the impurity. We shall single out these two channels from the free part of the Hamiltonian to obtain an effective theory by replacing Eq. (2.13) into Eq. (2.16) 


$$
\begin{aligned}
H_{e f f}= & \int_{0}^{\infty} \frac{p d p}{2 \pi} v_{F}|p|\left[\zeta_{0,+}^{\dagger}(p) \zeta_{0,+}(p)-\zeta_{0,-}^{\dagger}(p) \zeta_{0,-}(p)\right] \\
& +\int_{0}^{\infty} \frac{p d p}{2 \pi} v_{F}|p|\left[\zeta_{-1,+}^{\dagger}(p) \zeta_{-1,+}(p)-\zeta_{-1,-}^{\dagger}(p) \zeta_{-1,-}(p)\right] \\
& +\frac{g}{2} \int_{0}^{\infty} \frac{p d p}{2 \pi} \int_{0}^{\infty} \frac{q d q}{2 \pi}\left[\zeta_{0,+}^{\dagger}(p) \tau^{a} \zeta_{0,+}(q)+\zeta_{0,-}^{\dagger}(p) \tau^{a} \zeta_{0,-}(q)\right. \\
& \left.+\zeta_{0,+}^{\dagger}(p) \tau^{a} \zeta_{0,-}(q)+\zeta_{0,-}^{\dagger}(p) \tau^{a} \zeta_{0,+}(q)\right] S^{a} \\
+ & \frac{g}{2} \int_{0}^{\infty} \frac{p d p}{2 \pi} \int_{0}^{\infty} \frac{q d q}{2 \pi}\left[\zeta_{-1,+}^{\dagger}(p) \tau^{a} \zeta_{-1,+}(q)+\zeta_{-1,-}^{\dagger}(p) \tau^{a} \zeta_{-1,-}(q)\right. \\
& \left.\quad-\zeta_{-1,+}^{\dagger}(p) \tau^{a} \zeta_{-1,-}(q)-\zeta_{-1,-}^{\dagger}(p) \tau^{a} \zeta_{-1,+}(q)\right] S^{a}
\end{aligned}
$$

By using the correspondence between the $\zeta$ 's and c's defined above we may write the Hamiltonian in a more compact form. Since we found that this model contains effectively two "flavors" of right movers, we define a flavor index $l=1, \ldots, N_{f}$ with $N_{f}=2$. The system has an effective $S U(2)$ "flavor" symmetry which originates in the unavoidable mixing of angular momentum waves by the impurity. Similarly, we will write down the spin index $\sigma=1, \ldots, N_{c}$ explicitly. The effective Hamiltonian is (repeated indices are summed)

$$
H_{e f f}=\int_{-\infty}^{\infty} \frac{d p}{2 \pi} E(p) c_{l \sigma}^{\dagger}(p) c_{l \sigma}(p)+\frac{g}{2}\left[\int_{-\infty}^{\infty} \frac{d p}{2 \pi} \sqrt{|p|} c_{l \sigma}^{\dagger}(p)\right] \tau_{\sigma \omega}^{a}\left[\int_{-\infty}^{\infty} \frac{d q}{2 \pi} \sqrt{|q|} c_{l \omega}^{\dagger}(q)\right] f_{\alpha}^{\dagger} \tau_{\alpha \beta}^{a} f_{\beta}
$$

where $a=1, \ldots, N_{c}^{2}-1$. The single particle excitation energy is $E(p)=v_{F} p$. Eq. (2.18) shows that the coupling between the impurity spin and the band electrons is momentum dependent.

We note here that there is an alternative representation of this model. It may be obtained by, instead of rescaling the fields by a factor of $\sqrt{|p|}$ as we have done here, defining a new momentum variable $k \equiv p^{2}$ (an later extending $k$ to $(-\infty,+\infty)$ ). In this form, the effective Hamiltonian becomes

$$
H_{\text {eff }}=\int_{-\infty}^{\infty} \frac{d k}{2 \pi} E(k) c_{l \sigma}^{\dagger}(k) c_{l \sigma}(k)+\frac{g}{2}\left[\int_{-\infty}^{\infty} \frac{d k}{2 \pi} c_{l \sigma}^{\dagger}(k)\right] \tau_{\sigma \omega}^{a}\left[\int_{-\infty}^{\infty} \frac{d k^{\prime}}{2 \pi} c_{l \omega}^{\dagger}\left(k^{\prime}\right)\right] f_{\alpha}^{\dagger} \tau_{\alpha \beta}^{a} f_{\beta}
$$

In this case $E(k) \equiv \operatorname{sgn}(k) \sqrt{k}$. In this representation, the interaction becomes local at the expense of a non-local kinetic energy. In fact these two representations are equivalent and 
are the only ones compatible with local (anti)-commutation relations for the $\Psi$-fields. This is the representation used by Withoff and Fradkin [9]. In this paper we will not use this representation of the model.

We may now proceed to study this theory with a functional integral formalism [0]. The Lagrangian, in imaginary time, is

$$
\begin{aligned}
\mathcal{L}(\tau)= & \int_{-\infty}^{\infty} \frac{d p}{2 \pi} c_{l \sigma}^{\dagger}(p)\left(\frac{\partial}{\partial \tau}+E(p)\right) c_{l \sigma}(p) \\
+ & \frac{J_{0}}{N_{c}}\left[\int_{-\infty}^{\infty} \frac{d p}{2 \pi} \sqrt{|p|} c_{l \sigma}^{\dagger}(p)\right] \tau_{\sigma \omega}^{a}\left[\int_{-\infty}^{\infty} \frac{d q}{2 \pi} \sqrt{|q|} c_{l \omega}(q)\right] f_{\alpha}^{\dagger} \tau_{\alpha \beta}^{a} f_{\beta} \\
& +f_{\sigma}^{\dagger}\left(\frac{\partial}{\partial \tau}-\hat{h}_{\sigma}\right) f_{\sigma}+\epsilon_{f}(\tau)\left(f_{\sigma}^{\dagger} f_{\sigma}-Q_{f}\right)
\end{aligned}
$$

In Eq.(2.20) we have defined $J_{0} \equiv g\left(N_{c} / 2\right)$. We have also included a magnetic field $\hat{h}$ which we will choose to be a diagonal matrix $\hat{h}_{\sigma}$ and it will be defined below. The Lagrange multiplier field $\epsilon_{f}(\tau)$ has been introduced to enforce the constraint of charge (occupancy) $Q_{f}$ at the impurity site. In principle, the partition function separates into a sum of subsectors each of which is characterized by an impurity occupancy $Q_{f}$.

Following the standard large- $N_{c}$ decoupling approach of Read and Newns [7], we write the spin operators in terms of fermions and find an effective four Fermi interaction. This interaction can be written in a simple form by making use of the well known identity, which holds for the generators of $S U\left(N_{c}\right)$

$$
\sum_{a=1}^{N_{c}^{2}-1} \tau_{\sigma \omega}^{a} \tau_{\alpha \beta}^{a}=N_{c} \delta_{\sigma \beta} \delta_{\omega \alpha}-\delta_{\sigma \omega} \delta_{\alpha \beta}
$$

A Hubbard-Stratonovich (H-S) transformation is now introduced to decouple the fermionic quartic term which arises from this expansion. Up to an integration over the H-S fields $\varphi_{l}(\tau)$ and $\varphi_{l}^{*}(\tau)$, Eq. (2.20) is equivalent to

$$
\begin{aligned}
\mathcal{L}^{\prime}(\tau) & =\int_{-\infty}^{\infty} \frac{d p}{2 \pi} c_{l \sigma}^{\dagger}(p)\left(\frac{\partial}{\partial \tau}+E(p)\right) c_{l \sigma}(p)+\int_{-\infty}^{\infty} \frac{d p}{2 \pi} \sqrt{|p|}\left(\varphi_{l}^{*}(\tau) f_{\sigma}^{\dagger} c_{l \sigma}(p)+\varphi_{l}(\tau) c_{l \sigma}^{\dagger}(p) f_{\sigma}\right) \\
& +\frac{N_{c}}{J_{0}} \sum_{l=1}^{N_{f}}\left|\varphi_{l}(\tau)\right|^{2}+f_{\sigma}^{\dagger}\left(\frac{\partial}{\partial \tau}-\hat{h}_{\sigma}+\epsilon_{f}(\tau)\right) f_{\sigma}-Q_{f} \epsilon_{f}(\tau)
\end{aligned}
$$

Eq.(2.22) can be rearranged, by field shifting and completing squares, in the form 


$$
\begin{gathered}
\mathcal{L}^{\prime}(\tau)=\int_{-\infty}^{\infty} \frac{d p}{2 \pi}\left[c_{l \sigma}^{\dagger}(p)+\varphi_{l}^{*}(\tau) f_{\sigma}^{\dagger}(\tau) \sqrt{|p|}\left(\frac{\partial}{\partial \tau}+E(p)\right)^{-1}\right]\left(\frac{\partial}{\partial \tau}+E(p)\right) \\
\left.+c_{l \sigma}(p)+\left(\frac{\partial}{\partial \tau}+E(p)\right)^{-1} \sqrt{|p|} \varphi_{l}(\tau) f_{\sigma}(\tau)\right] \\
+f_{\sigma}^{\dagger}(\tau)\left[\frac{\partial}{\partial \tau}-\hat{h}_{\sigma}+\epsilon_{f}(\tau)-\varphi_{l}^{*}(\tau) \int_{-\infty}^{\infty} \frac{d p}{2 \pi}|p|\left(\frac{\partial}{\partial \tau}+E(p)\right)^{-1} \varphi_{l}(\tau)\right] f_{\sigma} \\
+\frac{N_{c}}{J_{0}} \sum_{l=1}^{N_{f}}\left|\varphi_{l}(\tau)\right|^{2}-Q_{f} \epsilon_{f}(\tau)
\end{gathered}
$$

We now obtain an effective theory for the fields $\epsilon_{f}$ and $\varphi_{l}$ by integrating out the fermions. The partition function is given by

$$
\begin{aligned}
Z & =\int D f^{\dagger} D f D c^{\dagger} D c D \epsilon_{f} D \varphi D \varphi^{*} \exp \left(-\int d \tau \mathcal{L}^{\prime}(\tau)\right) \\
& =Z_{0} \int D \varphi D \varphi^{*} D \epsilon_{f} \exp \left(-S_{\text {eff }}\right)
\end{aligned}
$$

where

$$
Z_{0}=\exp \left[N_{c} N_{f} \operatorname{Tr} \int \frac{d p}{2 \pi} \log \left(\frac{\partial}{\partial \tau}+E(p)\right)\right]
$$

is the partition function of free fermions. The impurity part of the effective action is

$$
\begin{aligned}
& S_{e f f}=-\sum_{\sigma=1}^{N_{c}} \operatorname{Tr} \log \left[\frac{\partial}{\partial \tau}-\hat{h}_{\sigma}+\epsilon_{f}-\sum_{l=1}^{N_{f}} \varphi_{l}^{*}(\tau) \int \frac{d p}{2 \pi}|p| \frac{1}{\partial_{\tau}+E(p)} \varphi_{l}(\tau)\right] \\
& +\int d \tau\left(\frac{N_{c}}{J_{0}}\left(\sum_{l=1}^{N_{f}}\left|\varphi_{l}\right|^{2}\right)-Q_{f} \epsilon_{f}\right)
\end{aligned}
$$

Here we stress that the model of physical interest has $N_{c}=2$ and $N_{f}=2$.

The effective action of Eq. (2.25) has the standard form of reference [7]. The key difference here is the form of the free fermion Green's function which in this problem has a relativistic form. For the usual Kondo problem the magnetic impurity is coupled to system of band electrons with a constant density of states at the Fermi surface. In the model that we discuss here, the density of states of the effective fermions (the "right movers") is still constant but the interaction with the magnetic impurity has an explicit momentum dependence. This momentum dependence is such that the effective coupling at low momenta becomes 
arbitrarily small. We will show below that up to a critical value of the coupling constant $J_{0}$ there is no Kondo effect. This is in fact the result of reference [9]. Notice that the momentum dependence of the interaction, is a direct consequence of the relativistic dispersion. Should any finite density of states arise, either by effects of a chemical potential or induced by disorder, a crossover to a conventional Kondo effect will occur. There is an important physical case in which a finite density of states is precluded by reasons of symmetry, and the Fermi energy has to be locked at zero. This is the case of the d-wave superconductors which we will discuss elsewhere [15].

We now consider the $N_{c} \rightarrow \infty$ limit. Here, large $N_{c}$ means the limit in which the rank of the group of spin rotations becomes large instead of being $S U(2)$. To proceed with the $1 / N_{c}$ expansion, we look first for static solution for $\varphi_{l}(\tau)$. From the $S U\left(N_{f}\right)$ flavor symmetry of the effective Hamiltonian, there is a manifold of solutions which span the group $S U\left(N_{f}\right)$. Clearly, all the solutions break $S U\left(N_{f}\right)$ spontaneously. As in all impurity problems, it is impossible to break spontaneously a continuous symmetry of bulk fermions by coupling them to an impurity, which has a finite Hilbert space. Thus, this apparent spontaneous symmetry breaking is an artifact of the $N_{c} \rightarrow \infty$ limit. In fact, we expect that it will already be restored by the leading $1 / N_{c}$ correction. This is precisely what happens in the large- $N$ approach to the conventional Kondo problem [7]. Thus, quantities which exhibit this apparent spontaneous symmetry breaking will get strongly corrected already in the next order in $1 / N_{c}$.

In what follows we will seek a static, symmetric, solution of the form $\varphi_{l}(\tau)=\varphi_{0}$. We will now derive the form of the Saddle Point equations which will determine $\varphi$ as a function of $J_{0}$ and of the filling fraction of the impurity $Q_{f}$.

First we need to compute

$$
g_{0}(i \omega)=-\int \frac{d p}{2 \pi}|p| \frac{1}{-i \omega+p}
$$

By working in imaginary frequency we automatically get the time-ordered expression form for Eq.(2.25). To compute Eq.(2.26) we introduce a lorentzian cutoff function $f_{\Lambda}(p)$ and 
extend the integration over $p$ to $\pm \infty$. We have

$$
g_{0}(i \omega)=-\int_{-\infty}^{\infty} \frac{d p}{2 \pi} \frac{|p|}{p-i \omega}\left(\frac{\Lambda^{2}}{p^{2}+\Lambda^{2}}\right)=\frac{i \omega}{2 \pi v_{F}^{2}} \frac{\Lambda^{2}}{\Lambda^{2}-\omega^{2}} \log \left(\frac{\omega^{2}}{\Lambda^{2}}\right)
$$

We can go back to Eq.(2.25) which now reads

$$
S_{e f f}=-N_{c} \beta \int_{0}^{\infty} \frac{d \omega}{2 \pi} \log \left[\epsilon_{f}^{2}+\omega^{2}\left(1-N_{f}\left|\varphi_{0}\right|^{2} f\left(\omega^{2}, \Lambda^{2}\right)\right)^{2}\right]+\beta\left(\frac{N_{c} N_{f}\left|\varphi_{0}\right|^{2}}{J_{0}}-Q_{f} \epsilon_{f}\right)
$$

where

$$
f\left(\frac{\omega^{2}}{\Lambda^{2}}\right)=\frac{1}{2 \pi v_{F}^{2}} \frac{1}{1-(\omega / \Lambda)^{2}} \log \left(\frac{\omega^{2}}{\Lambda^{2}}\right)
$$

and the magnetic field $h$ has been set to zero. The saddle point equations are

$$
N_{c} \int_{0}^{\infty} \frac{d \omega}{2 \pi} \frac{2 \epsilon_{f}}{\epsilon_{f}^{2}+\omega^{2}\left(1-N_{f}\left|\varphi_{0}\right|^{2} f\left(\omega^{2}, \Lambda^{2}\right)\right)^{2}}+Q_{f}=0
$$

where we should understand the integral as computed using some convenient adiabatic cutoff. The non-trivial solution for $\varphi_{0}$ is given by

$$
\frac{1}{J_{0}}=-\int_{0}^{\infty} \frac{d \omega}{2 \pi} \frac{2 \omega^{2} f\left(\omega^{2}, \Lambda^{2}\right)\left(1-N_{f}\left|\varphi_{0}\right|^{2} f\left(\omega^{2}, \Lambda^{2}\right)\right)}{\epsilon_{f}^{2}+\omega^{2}\left(1-N_{f}\left|\varphi_{0}\right|^{2} f\left(\omega^{2}, \Lambda^{2}\right)\right)^{2}}
$$

It will prove useful to define the dimensionless variables $x \equiv \frac{\omega}{\Lambda}$ and $\nu \equiv \frac{\left|\epsilon_{f}\right|}{\Lambda}$. The saddle point equations Eq.(2.30) and Eq.(2.31) now read

$$
\frac{Q_{f}}{N_{c}}=-\operatorname{sgn}\left(\epsilon_{f}\right) \int_{0}^{\infty} \frac{d x}{\pi} \frac{\nu}{\nu^{2}+x^{2}\left[1-\Delta \frac{\log x}{1-x^{2}}\right]^{2}}
$$

and

$$
\frac{1}{J_{0}}=-\frac{\Lambda}{\left(\pi v_{F}\right)^{2}} \int_{0}^{\infty} d x \frac{\log x}{1-x^{2}}\left(1-\Delta \frac{\log x}{1-x^{2}}\right) \frac{x^{2}}{\nu^{2}+x^{2}\left(1-\Delta \frac{\log x}{1-x^{2}}\right)^{2}}
$$

where $\Delta \equiv N_{f}\left|\varphi_{0}\right|^{2} / \pi v_{F}^{2}$.

There exists a critical value for $J_{0}$, which we define as the value of $J_{0}$ at the point where $\varphi_{0}$ departs from zero, for vanishing $\epsilon_{f}[9]$. It is given by

$$
\frac{1}{J_{c}}=-\int_{0}^{\infty} \frac{d \omega}{\pi} f\left(\frac{\omega^{2}}{\Lambda^{2}}\right)=-\frac{\Lambda}{\left(\pi v_{F}\right)^{2}} \int_{0}^{\infty} \frac{d x}{1-x^{2}} \log x=\frac{\Lambda}{\left(\pi v_{F}\right)^{2}} \frac{\pi^{2}}{4}
$$

In the next section we use the SPE to extract the critical behavior of the system near $J_{c}$. 


\section{SCALING AND ENERGY SCALES IN THE STATIC APPROXIMATION}

In the previous section we obtained the saddle-point equations (SPE) for this theory; however, these SPE have a singular behavior around the point $\nu=0, \Delta=0$. In particular, an expansion in powers of $\Delta$ for small $\Delta$ is not possible for $\nu \rightarrow 0$. In this section we want to investigate in further detail the behavior of the SPE and the scaling behavior of $\Delta$ as we approach the critical point. We will find that, opposite to the situation in the usual Kondo problem, there are now two independent energy scales for $\Delta$ and $\nu$.

We go back to the SPE expressed in their original form

$$
-\frac{Q_{f}}{N_{c}} \operatorname{sgn}\left(\epsilon_{f}\right)=\frac{1}{\pi} \int_{0}^{\infty} d x \frac{\nu}{\nu^{2}+x^{2}\left(1-\Delta \frac{\log x}{1-x^{2}}\right)^{2}}
$$

and

$$
\frac{1}{g_{0}}=-\int_{0}^{\infty} d x \frac{\log x}{1-x^{2}}\left(1-\Delta \frac{\log x}{1-x^{2}}\right) \frac{x^{2}}{\nu^{2}+x^{2}\left(1-\Delta \frac{\log x}{1-x^{2}}\right)^{2}}
$$

where $\Delta \equiv N_{f}\left|\varphi_{0}\right|^{2} / \pi v_{F}^{2}$ and $g_{0} \equiv \Lambda J_{0} /\left(\pi v_{F}\right)^{2}$.

In Eq.(3.1), the integrand is the equal time propagator for the impurity, with the integration variable $x$ being the imaginary frequency scaled by the band electrons cutoff $\Lambda$. This equation sets up a scale for a crossover between two different behaviors, in much the same way as in the usual Kondo effect the imaginary part of the band electrons Green function sets the scale of the Kondo temperature.

In the case of the usual Kondo effect, the expression equivalent to Eq.(3.1) is

$$
-\frac{Q_{f}}{N_{c}} \operatorname{sgn}\left(\epsilon_{f}\right)=\frac{1}{\pi} \int_{0}^{\infty} d x \frac{\nu}{\nu^{2}+(x+\Delta)^{2}}
$$

The case $N_{c}=2$ is, in a sense, a limiting case as can be seen from Eq.(3.3). On one hand, the r. h. s. of this equation is a positive function. This fact forces $\operatorname{sgn}\left(\epsilon_{f}\right)$ to be negative in order to have a solution. However, even in this case one gets

$$
Q_{f} \frac{\pi}{2}=\arctan \left(\frac{\Lambda}{\left|\epsilon_{f}\right|}\right)-\arctan \left(\frac{\Delta}{\left|\epsilon_{f}\right|}\right)
$$


where $\Lambda$ is some electron band cutoff. This suggests that for $Q_{f}=0$, the impurity level $\left|\epsilon_{f}\right|$ has to approach zero. However, if $Q_{f}=1$, there is no solution, since $\pi / 2-\arctan \left(\Lambda /\left|\epsilon_{f}\right|\right)$ is a strictly positive number. Now, if $N_{c}$ is an integer bigger than 2 , and letting $\Lambda \rightarrow \infty$, we find

$$
\pi\left(\frac{1}{2}-\frac{Q_{f}}{N_{c}}\right)=\arctan \left(\frac{\Delta}{\left|\epsilon_{f}\right|}\right)
$$

Here we can distinguish two different regimes: $Q_{f} / N_{c} \rightarrow 1 / 2$ which corresponds to the case $\Delta<<\left|\epsilon_{f}\right|$, and $Q_{f} / N_{c} \rightarrow 0$ which corresponds to $\left|\epsilon_{f}\right|<<\Delta$.

However, for the system being discussed here, the situation is a little different and, actually, more complex since, as it turns out, now there are two different scales involved. In principle there is a scale set on $x$ by $\Delta$ at the point where

$$
1 \approx-\Delta \frac{\log x}{1-x^{2}} \quad \text { or, } \quad \frac{1}{\Delta} \approx-\frac{\log x}{1-x^{2}}
$$

If the value of the frequency (and therefore of $x$ ) is small enough so that $x^{2} \ll 1$, we can approximate

$$
x \approx \exp \left(-\frac{1}{\Delta}\right)
$$

For $\Delta$ small enough, the approximation is consistent. Working on this idea, we split Eq.(3.1) as

$$
\begin{aligned}
\frac{Q_{f}}{N_{c}} & =\frac{1}{\pi} \int_{0}^{e^{-1 / \Delta}} d x \frac{\nu}{\nu^{2}+x^{2}\left(1-\Delta \frac{\log x}{1-x^{2}}\right)^{2}}+\frac{1}{\pi} \int_{e^{-1 / \Delta}}^{\infty} d x \frac{\nu}{\nu^{2}+x^{2}\left(1-\Delta \frac{\log x}{1-x^{2}}\right)^{2}} \\
& \equiv I_{1}+I_{2}
\end{aligned}
$$

where we have used the fact that the only solution consistent with $Q_{f} / N_{c}<1 / 2$ has $\epsilon_{f}<0$.

The SPE for $\Delta$ may be treated using a similar approach. Eq.(3.2) can be split into $I_{1}^{\prime}$ and $I_{2}^{\prime}$, where the first one is the corresponding integral up to $e^{-1 / \Delta}$, and the second one takes over from that point to infinity. Again we are interested in the small $\Delta$ and small $\nu$ regime. The detailed computation of the integrals $I_{1}, I_{2}$, and $I_{1}^{\prime}, I_{2}^{\prime}$ is given in appendix A.

We will be interested in the following limiting cases: 
a) $\frac{Q_{f}}{N_{c}}<<\frac{1}{\pi}$ :

In the regime in which $\nu<<e^{-1 / \Delta}<<1$, with $\nu, \Delta<<1$, the contribution from $I_{2}$ is neglegible and $\frac{Q_{f}}{N_{c}} \approx \frac{\nu}{\pi} e^{1 / \Delta}$ or $\nu \approx \pi \frac{Q_{f}}{N_{c}} e^{-1 / \Delta}<<e^{-1 / \Delta}$. Thus, in this regime,

$\frac{Q_{f}}{N_{c}}<<\frac{1}{\pi}$. In this limit, the leading term from the other SPE is going to be (see Appendix A, Eq.(A17))

$$
\left(\frac{\pi^{2}}{4}-\frac{1}{g_{0}}\right) \approx e^{-1 / \Delta}\left[\left(\pi \frac{Q_{f}}{N_{c}}\right)^{2} \frac{1}{\Delta}\right]
$$

This gives

$$
\Delta \approx \frac{1}{\log \left[\frac{\left(\pi \frac{Q_{f}}{N_{c}}\right)^{2}}{\frac{\pi^{2}}{4}-\frac{1}{g_{0}}}\right]}-\frac{\log \log \left[\frac{\left(\pi \frac{Q_{f}}{N_{c}}\right)^{2}}{\frac{\pi^{2}}{4}-\frac{1}{g_{0}}}\right]}{\log ^{2}\left[\frac{\left(\pi \frac{Q_{f}}{N_{c}}\right)^{2}}{\frac{\pi^{2}}{4}-\frac{1}{g_{0}}}\right]}+\ldots
$$

and

$$
\nu \approx \pi \frac{Q_{f}}{N_{c}} e^{-1 / \Delta} \approx \frac{\left(\frac{\pi^{2}}{4}-\frac{1}{g_{0}}\right)}{\pi \frac{Q_{f}}{N_{c}}} \frac{1}{\log \left[\frac{\left(\pi \frac{Q_{f}}{N_{c}}\right)^{2}}{\frac{\pi^{2}}{4}-\frac{1}{g_{0}}}\right]}
$$

b ) $\frac{Q_{f}}{N_{c}} \approx \frac{1}{2}$ :

We now consider the opposite regime, $e^{-1 / \Delta}<<\nu<<1$, where we obtain

$$
\frac{Q_{f}}{N_{c}} \approx \frac{1}{2}-\frac{3}{\pi \nu^{3}} e^{-3 / \Delta} \text { and } \nu \approx\left(\frac{3}{\pi}\right)^{1 / 3} \frac{e^{-1 / \Delta}}{\left(-\frac{Q_{f}}{N_{c}}+\frac{1}{2}\right)^{1 / 3}}>>e^{-1 / \Delta} \text { if } \frac{Q_{f}}{N_{c}} \rightarrow \frac{1}{2}
$$

In this regime, clearly $\frac{Q_{f}}{N_{c}} \rightarrow \frac{1}{2}$ and the other SPE gives

$$
\frac{1}{g_{0}} \approx \frac{\pi^{2}}{4}+\frac{\pi}{2} \nu \log \nu
$$

We can get a solution for $\nu$ by iteration on Eq.(3.13), 


$$
\nu \approx \frac{1}{\log \left[\frac{\pi}{2\left(\frac{\pi^{2}}{4}-\frac{1}{g_{0}}\right)}\right]} \frac{2}{\pi}\left(\frac{\pi^{2}}{4}-\frac{1}{g_{0}}\right)
$$

Using Eq.(3.12) we get $\nu$ which, after being replaced into Eq.(3.13) gives a scaling form for $\Delta$ in this regime

$$
\frac{2}{\pi}\left(\frac{\pi^{2}}{4}-\frac{1}{g_{0}}\right)\left[\frac{\pi}{3}\left(\frac{1}{2}-\frac{Q_{f}}{N_{c}}\right)\right]^{1 / 3} \approx e^{-1 / \Delta} \log \left[\frac{\pi}{2\left(\frac{\pi^{2}}{4}-\frac{1}{g_{0}}\right)}\right]
$$

Thus,

$$
\Delta \approx\left[\log \left[\frac{1}{\frac{2}{\pi}\left(\frac{\pi^{2}}{4}-\frac{1}{g_{0}}\right)\left(\frac{\pi}{3}\left(\frac{1}{2}-\frac{Q_{f}}{N_{c}}\right)\right)^{1 / 3}}\right]\right]^{-1}\left(1-\frac{\log \log \left[\frac{2}{\pi}\left(\frac{\pi^{2}}{4}-\frac{1}{g_{0}}\right)\right]^{-1}}{\log \left[\frac{2}{\pi}\left(\frac{\pi^{2}}{4}-\frac{1}{g_{0}}\right)\left(\frac{\pi}{3}\left(\frac{1}{2}-\frac{Q_{f}}{N_{c}}\right)\right)^{1 / 3}\right]^{-1}}\right)
$$

Notice that, for the physical case $N_{c}=2$, the two regimes $Q_{f} / N_{c}<<1$ and $Q_{f} \approx N_{c} / 2$ are identical. Only as $N_{c}$ grows large it becomes possible to distinguish one case from the other. However, in the case $Q_{f} \approx N_{c} / 2$ the magnitude of $\Delta$ appears to go to zero as $Q_{f} \rightarrow N_{c} / 2^{-}$ even though the critical coupling is independent of the value of $Q_{f}$. In contrast, in the case $Q_{f} / N_{c}<<1$ such unwanted feature is not present. It is worth to note here that, in spite of this apparent difficulty, we will show at the end of this section that the zero-temperature suceptibility at zero field has the same behavior in both regimes. Thus, this difficulty is not physically relevant. For simplicity, we will use the case $Q_{f} / N_{c}<<1$ to extrapolate to the physically meaningful case of $N_{c}=2$.

We can use these results to derive the $\beta$-function for the coupling constant $g_{0}$ in the limit $N_{c} \rightarrow \infty$. In order to do this we may use Eq.(3.13) for the regime in which $\frac{Q_{f}}{N_{c}} \approx \frac{1}{2}$, or Eq.(3.11) for the case $\frac{Q_{f}}{N_{c}} \rightarrow 0$. Starting from Eq.(3.9) we may replace $\Delta$ in terms of $\nu$ to obtain

$$
\frac{\pi^{2}}{4}-\frac{1}{g_{0}} \approx \alpha \nu(\log \alpha-\log \nu)
$$

where $\alpha \equiv \pi \frac{Q_{f}}{N_{c}}$. The $\beta$-function keeps track of the flow of $g_{0}$ as the cutoff $\Lambda$ is decreased from very large values (infinite bandwidth). Hence, 


$$
\beta\left(g_{0}\right) \equiv-\Lambda \frac{\partial g_{0}}{\partial \Lambda}
$$

In both limits $Q_{f} / N_{c} \rightarrow 0$ and $Q_{f} / N_{c} \rightarrow 1 / 2$ we find

$$
\beta\left(g_{0}\right)=-g_{0}+\frac{1}{g_{c}} g_{0}^{2}
$$

where $\frac{1}{g_{c}}=\frac{\pi^{2}}{4}$.

We immediately see that there are two fixed points: (a) a stable fixed point at $g_{0}=0$ and (b) an unstable fixed point at $g_{0}=g_{c}$. The stable fixed point represents the weak coupling phase in which the fermions are decoupled from the impurity and the impurity spin is unscreened and there is no Kondo effect. The non-trivial fixed point at $g_{0}=g_{c}$ separates the weak coupling phase from a strong coupling phase that, in principle, should exhibit a Kondo effect. This unstable fixed point can be regarded as the usual marginally unstable fixed point of the standard Kondo problem, now pushed to a finite value of the coupling constant by effect of the reduction of the density of band states. Hence, in the present case, the interaction has to become strong enough so as to overcome the effect of the depletion in the number of states available in order to produce the Kondo screening. Thus, for systems with a small coupling to the impurity, there will be no Kondo screening and we should expect a decoupled impurity behavior.

If $g_{0}>g_{c}$, the system is in the Kondo screeening phase. We may define a Kondo scale $T_{K}$ for the regime $g_{0}>g_{c}$. From Eq.(3.9) through Eq.(3.11) we see that, in terms of the physical parameters of the theory we have

$$
T_{K} \equiv\left|\epsilon_{f}\right| \approx \pi \frac{N_{c}}{Q_{f}} v_{F}^{2}\left(\frac{J_{0}-J_{c}}{J_{0} J_{c}}\right)\left(\log \left[\left(2 \frac{Q_{f}}{N_{c}}\right)^{2}\left(\frac{J_{0}}{J_{0}-J_{c}}\right)\right]\right)^{-1}
$$

The results found here generalize the work of WF [9]. In that work the case of a density of states which vanishes linearly was not discussed on account that the structure of the singularities was not smoothly connected with the case of a constant density of states. The results of this section show that the main difference is the presence of logarithmic corrections to scaling in all the physical quantities. The case of a magnetic impurity coupled to a Fermi 
system with a linear density of states seems to be analogous to the behavior of critical systems at their upper critical dimension. In contrast, the case of the constant density of states is the analog of a critical system at the lower critical dimension (marginal instability). The closest analog to this problem is the critical behavior of the Gross-Neveu Model in $3+1$ (space-time) dimensions [20].

The results that we find here hold for the simple regime of large $N_{c}$ which does not describe correctly the dynamics of the system with more than one fermion flavor. At this leading order in $1 / N_{c}$, the existence of several channels is only reflected into a trivial degeneracy and it does not lead to any new physics. However, as has been shown by Blandin and Nozières [18], already the leading corrections in $1 / N_{c}$ will lead to non-trivial behavior in the "screened" phase, $g_{0}>g_{c}$. In the standard Kondo problem, this behavior was confirmed by both the Bethe-Ansatz solution [17] and Conformal Field Theory [21]. We note here that, due to the non-local character of the effective one-dimensional theory discussed in section [I], it is not possible to use neither the Bethe-Ansatz nor Conformal Field Theory to study the non-critical Kondo problem that we discuss here. Nevertheless, we expect that the physics of the phase with $g_{0}>g_{c}$ should be similar to that of the conventional overscreend Kondo problem. We also note that, in a more realistic model, one expects four nodes with two nodes of each chirality and, hence with more flavors and a more pronounced overscreened behavior.

We close this section with a discussion of the zero-field susceptibility for this model. The coupling of a magnetic field only to the impurity spin has the form of a Zeeman term

$$
H_{h}=-\sum_{\sigma=1}^{N_{c}} f_{\sigma}^{\dagger} \hat{h}_{\sigma} f_{\sigma}
$$

Here, $\hat{h}_{\sigma}$ are the elements of a suitably chosen $S U\left(N_{c}\right)$ diagonal generator, which also includes the appropriate normalization factors so as to set the Bohr magneton to one. We will need to ensure the tracelessness of the generator, and we can do so by keeping all the elements of order one with alternating signs. In this way we prevent the case of overpopulating one of the states that the Zeeman term splits with respect to the others, i.e., we explicitly avoid 
cases like the one with $N_{c}-1$ elements being -1 and the last element being $N_{c}-1$. This is an important consideration to care about if we want to study the magnetic field crossover, i.e., if we were interested in obtaining the suceptibility at finite field. This was discussed in reference [22]. However, we will be interested only in the zero-field suceptibility and it will be sufficient to assume all the $\hat{h}_{\sigma}$ to be $\pm h$, so that the sum of their squares gives a factor of $N_{c}$. The magnetization $M$ is given by

$$
M=\sum_{\sigma=1}^{N_{c}}\left\langle f_{\sigma}^{\dagger} \hat{h}_{\sigma} f_{\sigma}\right\rangle=-\sum_{\sigma=1}^{N_{c}} \int_{-\infty}^{\infty} \frac{d \omega}{2 \pi} \frac{\hat{h}_{\sigma}}{\epsilon_{f}-i \omega\left(1-N_{f}\left|\varphi_{0}\right|^{2} f\left(\omega^{2}, \Lambda^{2}\right)\right)-\hat{h}_{\sigma}}
$$

The susceptibility at zero field is

$$
\left.\chi\right|_{h=0}=\left.\frac{\partial M}{\partial h}\right|_{h=0}=\frac{N_{c}}{\Lambda}\left\{\frac{2}{\nu}-\frac{\partial}{\partial \nu}\right\} \int_{0}^{\infty} \frac{d x}{\pi} \frac{\nu}{\nu^{2}+x^{2}\left(1-\Delta \frac{\log x}{1-x^{2}}\right)^{2}}
$$

Once again, we consider two regimes: $Q_{f} / N_{c} \rightarrow 0$ and $Q_{f} / N_{c} \rightarrow 1 / 2$. We discuss only the regime $g_{0}>g_{c}$ since the suceptibility is infinite below $g_{c}$, where the impurity is unscreened.

a ) $\frac{Q_{f}}{N_{c}} \ll 1$ :

For $\frac{Q_{f}}{N_{c}} \approx \frac{\nu}{\pi} e^{1 / \Delta}$, we get

$$
\chi(0) \approx \frac{Q_{f}}{\left|\epsilon_{f}\right|}=\frac{Q_{f}}{T_{K}} \approx \frac{N_{c}}{\pi v_{F}^{2}}\left(\frac{Q_{f}}{N_{c}}\right)^{2}\left(\frac{J_{c} J_{0}}{J_{0}-J_{c}}\right) \log \left[\left(\frac{2 Q_{f}}{N_{c}}\right)^{2}\left(\frac{J_{0}}{J_{0}-J_{c}}\right)\right]
$$

b ) $\frac{Q_{f}}{N_{c}} \approx \frac{1}{2}$ :

For $\frac{Q_{f}}{N_{c}} \approx \frac{1}{2}-\frac{3}{\pi \nu^{3}} e^{-3 / \Delta}$, we have

$$
\chi(0) \approx \frac{N_{c}}{2 \pi v_{F}^{2}}\left(\frac{J_{c} J_{0}}{J_{0}-J_{c}}\right) \log \left[\frac{2}{\pi}\left(\frac{J_{0}}{J_{0}-J_{c}}\right)\right]\left\{1-5\left(\frac{1}{2}-\frac{Q_{f}}{N_{c}}\right)+\ldots\right\}
$$

Once again, we find logarithmic corrections to scaling.

We see from Eq.(3.25) and Eq.(3.24) that the zero-field susceptibility $\chi(0)$, at zero temperature, has a behavior consistent with the general picture described above. It is finite for $g_{0}>g_{c}$, but diverges as $g_{0}$ approaches $g_{c}$. This is consistent with the impurity being screeened (Kondo effect) for a value of the coupling constant bigger than the critical value. 
However, as we approach the critical coupling constant from the screening (Kondo) phase, the susceptibility must diverge, since for values of the coupling lower than the critical value, $\Delta$ vanishes and the impurity effectively decouples from the band. In this regime one expects the impurity will behave like a free spin in a magnetic field and, since we are at zero temperature, the susceptibility should diverge.

\section{T-MATRIX AND ONE PARTICLE GREEN FUNCTION}

In this section we derive the propagator for the band electrons to order $N \rightarrow \infty$. We will use this expression to calculate the $T$-matrix in $N \rightarrow \infty$ limit. We will consider processes in which an electron with a given energy is initially in a state with well defined angular momentum, and it is scattered by the impurity into a state with possibly a different angular momentum. Thus, we will parametrize the $T$-matrix by the magnitude of the incoming and the outgoing momenta (namely, the energy of the state) and by the angular momenta of channels involved in the scattering process. This description is natural since at most only a pair of angular moementum states $(\ell=0,-1$ with our choice of node) are actually mixed by the impurity.

In contrast, the computation of the correlation function $\left\langle\Psi_{j}^{\dagger}(\vec{r}) \Psi_{k}\left(\vec{r}^{\prime}\right)\right\rangle$, for $\vec{r}$ and $\vec{r}^{\prime}$ away from the impurity will involve a mixing of all the angular momentum channels. However, in principle, we could add a source term and also decompose the source field in angular momentum modes, and keep only the modes that get mixed by the interaction with the impurity. In other words, we are considering the scattering of band electrons among angular momentum channels produced by the interaction with the impurity. In the case of a coupling to a flat band (usual Kondo problem), the only angular momentum channel that gets involved is the $s$-wave. Thus, in practice, we only need to consider the partial waves that actually get mixed by scattereing from the impurity.

Therefore, it will be sufficient to work with the equivalent 1-dimensional fermions $c_{1}$ and

$c_{2}$ while keeping in mind the correspondences defined in section $\mathbb{\Psi}$. The relevant part of the 
action is

$$
\begin{gathered}
S_{F}=\int_{-\infty}^{\infty} \frac{d \omega}{2 \pi} \int_{-\infty}^{\infty} \frac{d p}{2 \pi} c_{l \sigma}^{\dagger}(p, \omega)(-i \omega+E(p)) c_{l \sigma}(p, \omega) \\
+\int_{-\infty}^{\infty} \frac{d \omega}{2 \pi} \int_{-\infty}^{\infty} \frac{d \omega^{\prime}}{2 \pi} \int_{-\infty}^{\infty} \frac{d p}{2 \pi} \sqrt{|p|}\left\{\varphi_{l}^{*}\left(\omega-\omega^{\prime}\right) f_{\sigma}^{\dagger}\left(\omega^{\prime}\right) c_{l \sigma}(p, \omega)\right. \\
\left.+\varphi_{l}\left(\omega-\omega^{\prime}\right) c_{l \sigma}^{\dagger}(p, \omega) f_{\sigma}\left(\omega^{\prime}\right)\right\}
\end{gathered}
$$

We add sources $\eta_{l \sigma}^{\dagger}(p, \omega)$ and $\eta_{l \sigma}(p, \omega)$ for the fields $c_{l \sigma}(p, \omega)$ and $c_{l \sigma}^{\dagger}$ by adding the following source term to the action

$$
\int_{-\infty}^{\infty} \frac{d \omega}{2 \pi} \int_{-\infty}^{\infty} \frac{d p}{2 \pi}\left(\eta_{l \sigma}^{\dagger}(p, \omega) c_{l \sigma}(p, \omega)+c_{l \sigma}^{\dagger}(p, \omega) \eta_{l \sigma}(p, \omega)\right)
$$

After completing squares and integrating over the band fermion fields, we are left with (appart from a normalization factor) an action which depends only on the impurity fermion fields and the sources,

$$
\begin{aligned}
S_{f} & =\sum_{\sigma=1}^{N_{c}} \int \frac{d \omega}{2 \pi} f_{\sigma}^{\dagger}(\omega)\left(-i \omega+\epsilon_{f}\right) f_{\sigma}(\omega) \\
& -\sum_{a, w} \int_{-\infty}^{\infty} \frac{d \omega}{2 \pi} \int_{-\infty}^{\infty} \frac{d p}{2 \pi}\left[\int \frac{d \omega^{\prime}}{2 \pi} \varphi_{l}^{*}\left(\omega-\omega^{\prime}\right) \sqrt{|p|} f_{\sigma}^{\dagger}\left(\omega^{\prime}\right)+\eta_{l \sigma}^{\dagger}(p, \omega)\right]\left(\frac{1}{-i \omega+E(p)}\right) \\
& +\int_{-\infty}^{\infty} \frac{d \omega}{2 \pi} \int_{-\infty}^{\infty} \frac{d p}{2 \pi} \eta_{l \sigma}^{\dagger}(p, \omega)\left(\frac{d \omega^{\prime \prime}}{2 \pi} \varphi_{l}^{*}\left(\omega-\omega^{\prime \prime}\right) \sqrt{|p|} f_{\sigma}\left(\omega^{\prime \prime}\right)+\eta_{l \sigma}(p, \omega)\right]
\end{aligned}
$$

After a few fairly straightforward manipulations and another integration now over the impurity fermion fields, we can re-write the action as

$$
S_{f}\left[J, J^{\dagger}\right]=-\sum_{\sigma=1}^{N_{c}} \int \frac{d \omega}{2 \pi} \int \frac{d \omega^{\prime}}{2 \pi} J_{\sigma}^{\dagger}(\omega) K^{-1}\left(\omega, \omega^{\prime}\right) J_{\sigma}\left(\omega^{\prime}\right)
$$

where we have introduced the operators $J_{\sigma}$ 's and the kernel $K$ which are given by

$$
J_{\sigma}(\omega)=\sum_{l=1}^{N_{f}} \int \frac{d \omega^{\prime}}{2 \pi} \int \frac{d p}{2 \pi} \varphi_{l}^{*}\left(\omega^{\prime}-\omega\right) \frac{\sqrt{|p|}}{-i \omega^{\prime}+E(p)} \eta_{l \sigma}\left(p, \omega^{\prime}\right)
$$

and

$$
K\left(\omega, \omega^{\prime}\right)=\left(-i \omega+\epsilon_{f}\right) \delta\left(\omega-\omega^{\prime}\right)-\int \frac{d p}{2 \pi} \int \frac{d \omega^{\prime \prime}}{2 \pi} \frac{|p|}{-i \omega^{\prime}+E(p)} \sum_{l=1}^{N_{f}} \varphi_{l}^{*}\left(\omega^{\prime \prime}-\omega\right) \varphi_{l}\left(\omega^{\prime \prime}-\omega^{\prime}\right)
$$


The generating functional for the Green's functions (at zero temperature) can be written as

$$
Z_{F}\left[\eta, \eta^{\dagger}\right]=\exp \left(-S_{f}\left(\eta, \eta^{\dagger}\right)\right)
$$

so that correlation functions of the band fermion fields can be computed by differentiation over $Z_{F}$

$$
\left\langle c_{l \sigma}^{\dagger}(q, \Omega) c_{l^{\prime} \sigma^{\prime}}\left(q^{\prime}, \Omega^{\prime}\right)\right\rangle=\left\langle\frac{\delta}{\delta \eta_{l \sigma}(q, \Omega)} \frac{\delta}{\delta \eta_{l^{\prime} \sigma^{\prime}}\left(q^{\prime}, \Omega^{\prime}\right)} Z_{F}\left[\eta, \eta^{\dagger}\right]\right\rangle_{\eta=\eta^{\dagger}=0}
$$

We restrict the calcultation to the static solution case where $\varphi_{l}(\Omega-\omega) \equiv \bar{\varphi}_{l} \delta(\Omega-\omega)$. Then

$$
\begin{gathered}
\left\langle c_{l \sigma}^{\dagger}(q, \Omega) c_{l^{\prime} \sigma^{\prime}}\left(q^{\prime}, \Omega^{\prime}\right)\right\rangle=\delta_{l l^{\prime}} \delta_{\sigma \sigma^{\prime}} \delta\left(q-q^{\prime}\right) \delta\left(\Omega-\Omega^{\prime}\right) \frac{1}{-i \Omega+E(q)} \\
+\frac{\sqrt{|q|}}{-i \Omega+E(q)} \mathcal{T}_{l l^{\prime}}(\Omega) \frac{\sqrt{\left|q^{\prime}\right|}}{-i \Omega+E\left(q^{\prime}\right)} \delta\left(\Omega-\Omega^{\prime}\right)
\end{gathered}
$$

where the $T$-matrix $\mathcal{T}_{l l^{\prime}}(\Omega)$ is given by

$$
\mathcal{T}_{l l^{\prime}}(\Omega) \equiv-\frac{\bar{\varphi}_{l} \bar{\varphi}_{l^{\prime}}^{*}}{-i \Omega+\epsilon_{f}+g_{0}(i \Omega) \sum_{l=1}^{N_{f}}\left|\bar{\varphi}_{l}\right|^{2}}
$$

where $g_{0}(i \Omega)$ was given in Eq. (2.26) and Eq. (2.27).

The $T$-matrix of eq. (4.10) exhibits several important features:

a ) Eq. (4.10) gives the $T$-matrix at imaginary frequency. The $T$-matrix for real frequency (at zero temperature) is found by an analytic continuation to the real axis, $i \Omega \rightarrow \Omega$.

b ) If $\bar{\varphi}_{l}$ and $\bar{\varphi}_{l^{\prime}}^{*}$ are non-zero, there exists a finite matrix element for the scattering of a band electron from a state with angular momentum $l$ into a state with angular momentum $l^{\prime}$ (and viceversa). In contrast, for a flat band, the $T$-matrix is diagonal in angular momentum states. This matrix element is not invariant under $S U\left(N_{f}\right)$ since it mixes the angular momentum channels. As we pointed out above, this is a consequance of the unphysical spontaneous breaking of the flavor (channel) symmetry. Thus only the trace of this T-matrix is physical. All other matrix elements will be suppressed by infrared divergent corrections to next order in the $1 / N_{c}$ expansion. 
c ) From the analytic continuation of the trace of the $T$-matrix one can find the position of the Kondo resonance $\Omega_{K}$, the width $W_{K}$ and the phase shift $\delta(\Omega)$. Using the fact that $\sum_{l=1}^{N_{f}}\left|\bar{\varphi}_{l}\right|^{2}=N_{f}\left|\varphi_{0}\right|^{2}$, we can write the trace in the form

$$
\operatorname{tr} \hat{\mathcal{T}}(\Omega) \equiv \mathcal{T}_{l l}(\Omega)=-\frac{\pi v_{F}^{2} \Delta}{\Omega+\left|\epsilon_{f}\right|+\Omega \Delta \log \frac{\Lambda}{|\Omega|}-i \frac{\pi}{2} \Omega \Delta}
$$

which is valid for frequencies low compared to the bandwidth $\Omega \ll \Lambda$ but comparable to the dynamically generated scales. The phase shift $\delta(\Omega)$ is

$$
\delta(\Omega)=\tan ^{-1}\left(\frac{\frac{\pi}{2} \Omega \Delta}{\Omega+\left|\epsilon_{f}\right|+\Omega \Delta \log \frac{\Lambda}{|\Omega|}}\right)
$$

d ) The imaginary part of the $T$-matrix is

$$
\operatorname{Im} \operatorname{tr} \hat{\mathcal{T}}(\Omega)=\frac{\frac{1}{2}\left(\pi \Delta v_{F}\right)^{2} \Omega}{\left(\left|\epsilon_{f}\right|+\Omega\left(1-\Delta \log \frac{|\Omega|}{\Lambda}\right)\right)^{2}+\left(\frac{\pi}{2} \Delta \Omega\right)^{2}}
$$

The function given by Eq.(4.13) has a peak at the Kondo resonance $\Omega_{K}$, which is the solution of the equation

$$
\epsilon_{f}=\Omega\left(1-\Delta \log \frac{|\Omega|}{\Lambda}\right)
$$

One easily obtains the asymptotic solution

$$
\Omega_{K} \approx-\pi v_{F}^{2} \frac{N_{c}}{Q_{f}}\left(\frac{J_{0}-J_{c}}{J_{0} J_{c}}\right) \frac{1}{\log \left[\frac{2}{\pi}\left(\frac{2 Q_{f}}{N_{c}}\right)^{3}\left(\frac{J_{0}}{J_{0}-J_{c}}\right)^{2}\right]}+\ldots
$$

where we have kept only the leading logarithmic corrections. We see immediately that we have a resonance centered at $\Omega_{K}$, which is basically the Kondo scale $\epsilon_{f}$ that we found in section $\mathbb{\text { III }}$ but modified by the ubiquitous logarithmic corrections. In particular, the position of the Kondo resonance scales with the distance to the critical coupling constant and it goes to zero (the Fermi energy) at $J_{c}$.

e ) The resonance width $W_{K}$ can be read off from Eq.(4.13)

$$
W_{K} \approx\left|\Omega_{K}\right| \frac{\pi \Delta}{2}
$$


From Eq. (4.12) it is clear that the dimensionless parameter $\Delta=N_{f}\left|\bar{\varphi}_{0}\right|^{2} / \pi v_{F}^{2}$ sets the scale for the range of the scattering amplitude. Thus, as far as transport properties such as the resistivity are concerned, $\Delta$ is the important parameter, whereas thermodynamic properties such as the suceptibility, depend entirely on the impurity energy scale $\epsilon_{f}$, i.e., the Kondo scale $T_{K}$. The analysis done in the last section shows that, due to the strong logarithmic corrections, these two scales are completely different. Once again, in the conventional Kondo effect, $T_{K}$ controls both effects.

Hence, in contrast with conventional Kondo behavior, the interaction with the magnetic impurity induces a non-trivial renormalization of the propagator of the band fermions. This will happen even for $J_{0} \leq J_{c}$, although this effect only occurs in higher order in $1 / N_{c}$. Finally we stress that, due to the $S U\left(N_{f}\right)$ flavor symmetry, there cannot be any channel mixing scattering processes in this model. However, in the following section, we will present a model which describes the most general impurity scattering processes for systems with only one node. In that model the channel (or flavor) symmetry $S U\left(N_{f}\right)$ is broken explicitly already at the level of the impurity Hamiltonian. Thus we expect to find processes which will mix the various channels.

\section{GENERALIZED IMPURITY}

In the past sections we considered a situation in which the fermions coupled to the impurity spin only through their own spin density $\Psi^{\dagger} \vec{\tau} \Psi$. Scattering processes of this type have the simplifying feature that particles and holes interact with the impurity independently from each other and exactly in the same way. However, in practice, more general scattering processes will be present. We also made the assumption that only one node of the twodimensional fermions is present.

Here, we will consider all possible scattering processes involving only one node. The coupling through the spin density has the very special feature that it is diagonal in the components of the spinors, i.e., the impurity does not mix particles with holes. In terms 
of the effective Hamiltonian of Eq. (2.19), this diagonal coupling implies that there is no explicit mixing of angular momentum channels. Hence, the model has an $S U\left(N_{f}\right)$ symmetry (with $N_{f}=2$ ). Any process which mixes particles and holes will break the flavor symmetry explicitly. We will include now these processes.

We will also incorporate the correct node structure. For example, in the case of a flux phase say, on a square lattice, there will be four different nodes corresponding to the symmetry points $\left( \pm \pi / 2 a_{0}, \pm \pi / 2 a_{0}\right)$ of the first Brillouin zone. As we discussed in section 四, a $d$-wave superconductor has an analogous node structure, at the points where the gap has nodes. We will discuss of this very interesting case in a separate publication [15]. It is straightforward to see that the main effect of a more general node structure is to increase the number of flavors in the effective model. In the one-node model of the previous section we found that there were two flavors and an $S U(2)$ flavor symmetry. The existence of two flavors can be traced back to the spinor structure of the original problem. When the two-dimensional fermions have more than one node, the number of flavors becomes twice the number of nodes, $N_{f}=2 N_{\text {nodes }}$. Thus, for a flux phase we will have $N_{f}=8$ flavors. However, flux phases are even under parity. Hence, there are two pairs of nodes with opposite parity. The parity of the node is given by the relative sign of the two terms in the free fermion Hamiltonian of Eq. (2.1). Thus, a change of parity is equivalent to the mapping $\theta \rightarrow-\theta$, in other words to a reflection across the $x_{1}$ axis. In Section 【1 we showed that, with the choice of parity we made there, the channels with angular momenta $\ell=0$ and $\ell=-1$ get mixed by the impurity. Hence, if nodes with the other parity are also included, we will also get mixing between $\ell=0$ and $\ell=+1$, but no direct mixing between $\ell=+1$ and $\ell=-1$. In Section $\mathbb{I}$ we also found that only the mapping between the components of the original fermion and the fermions of the effective one-dimensional theory carried information about which angular momentum components are mixed. This feature is always present. Thus, the net effect of having a multinode structure is to increase the number of flavors. Consequently, although the thermodynamic properties will be insensitive to parity assignments, the scattering amplitudes of the original fermions with the impurity will carry 
the information of the parity of the nodes. This feature can be used to determine the nature of a superconducting state.

In what follows, we will consider a system with four nodes, two with positive parity and two with negative parity, interacting with a single magnetic impurity. For simplicity we will assume that the impurity form factor is strongly peaked at small momentum, so that inter-node scattering processes can be ignored. We will keep, however, processes in which particles and holes may interact differently with the impurity and/or mix with each other. We parametrize these processes with four coupling constants $J_{i}$, with $i=0,1,2,3$. The generalized impurity Hamiltonian is given by

$$
H_{i m p}=\sum_{l, i} J_{i} \Psi_{\alpha l}^{\dagger}(0) T_{i} \vec{\tau} \Psi_{\beta l}(0) \vec{S}
$$

where the indices $l$ labels the nodes and $\alpha$ and $\beta$ the spin components. $T_{0}=I$ is the $2 \times 2$ identity matrix and $T_{i}=\sigma_{i}(i=1,2,3)$ are the three Pauli matrices. Here $J_{0}$ is the coupling constant that was used in the model of Section [1].

The free fermion Hamiltonian for each node

$$
H_{0}=\int \frac{d^{2} p}{(2 \pi)^{2}} \Psi^{\dagger}(p) v_{F} \vec{\sigma} \cdot \vec{p} \Psi(p)
$$

is invariant under the symmetry transformation $\mathrm{C} \Psi(p) \rightarrow \sigma_{3} \Psi(-p)$. The effect of this transformation on on $H_{\text {imp }}$ is to reverse the sign of both $J_{1}$ and $J_{2}$. This implies that the sign of $J_{1}$ and $J_{2}$ is irrelevant to the properties of the theory, which should depend only on the absolute values of these two coupling constants. In the expressions for the Fermi field

$$
\Psi_{i l}(0)=\frac{1}{\sqrt{2}} \int_{-\infty}^{\infty} \frac{d p}{2 \pi} \sqrt{|p|} c_{i l}(p) \quad \text { and } \quad S^{a} \equiv f_{\gamma}^{\dagger} \tau_{\gamma \delta}^{a} f_{\delta}
$$

we have $l=1, \ldots, N_{\text {nodes }}$ and $i=1,2$. In what follows we use a single flavor index $i=$ $1, \ldots, N_{f}$ with $N_{f}=2 N_{\text {nodes }}$. However, we will keep in mind that the impurity does not mix different nodes but it does mix the flavor components associated with the same node.

The model of equivalent one-dimensional right-moving excitations has an $S U(2)$ spin (color) symmetry and an $S U\left(2 N_{\text {nodes }}\right)$ flavor symmetry (associated with the channels) broken down to $S U\left(N_{\text {nodes }}\right)$. The free fermion kinetic term has the form 


$$
H_{0}=\int \frac{d p}{2 \pi} p v_{F} \sum_{i \alpha} c_{i \alpha}^{\dagger}(p) c_{i \alpha}(p)
$$

and an impurity interaction term with the following structure

$$
H_{i m p}=-\int \frac{d p}{2 \pi} \int \frac{d q}{2 \pi} \sqrt{|p||q|} f_{\alpha}^{\dagger} c_{\alpha j}(q) c_{\beta i}^{\dagger}(p) f_{\beta} T_{i j}
$$

where $T_{i j}$ is the coupling matrix which has a block diagonal form. For $i, j$ associated with the same node $T_{i j}$ has the form

$$
T_{i j} \equiv N_{c}\left(J_{0} \delta_{i j}+J_{1} \sigma_{1}^{i j}+J_{2} \sigma_{2}^{i j}+J_{3} \sigma_{3}^{i j}\right)
$$

In other terms the matrix has the form $T \otimes I$ where $T$ is the $2 \times 2$ matrix of Eq. (5.6) and $I$ is the $N_{\text {nodes }} \times N_{\text {nodes }}$ identity matrix. We have also let the spin indices $\alpha, \beta$ to run from $1, \ldots, N_{c}$ and used the identity of Eq. (2.21). The form of the impurity Hamiltonian Eq. (5.5) shows that, if the coupling constants $J_{i}$ are all different, the $S U(2)$ flavor symmetry of each node is broken by the interactions but the symmetry involving different nodes remains intact.

We will now proceed as in the previous sections and solve this model in the limit of $N_{c} \rightarrow \infty$. After a Hubbard-Stratonovich transformation, the quartic term of the euclidean action becomes (repeated indices are summed)

$$
\int d \tau \sigma_{i}^{*}(\tau) M_{i j} \sigma_{j}(\tau)+\int d \tau \int_{-\infty}^{\infty} \frac{d p}{2 \pi} \sqrt{|p|}\left(\sigma_{i}(\tau) f_{\alpha}^{\dagger}(\tau) c_{\alpha i}(p, \tau)+\sigma_{i}^{*}(\tau) c_{\alpha i}^{\dagger}(p, \tau) f_{\alpha}(\tau)\right)
$$

where $M_{\alpha \beta}=\left(T_{\alpha \beta}\right)^{-1}$. Since $T$ is of the form $T \otimes I$, then $M$ has the same form, i.e., $M \otimes I$. In the following, $M$ stands for the $2 \times 2$ matrix of Eq. (5.7). It can be easily shown that

$$
M=\frac{N_{c}}{g_{0}^{2}-\vec{g} \cdot \vec{g}}\left(\begin{array}{lr}
g_{0}-g_{3} & -\left(g_{1}-i g_{2}\right) \\
-\left(g_{1}+i g_{2}\right) & g_{0}+g_{3}
\end{array}\right) \equiv N_{c} \tilde{M}
$$

where $J_{i} \equiv g_{i} / N_{c}^{2}$ and $\vec{g} \equiv\left(g_{1}, g_{2}, g_{3}\right)$. In the case considered in section $\llbracket$, i.e., when $J_{i}=0$, $J_{0} \neq 0$, we have a full $U(2)$ flavor symmetry between the $\sigma$-fields. In other words, the two channels play exactly the same role. When the coupling constants are all different, this symmetry is broken down to a $U(1) \times U(1)$, where one of the $U(1)$ symmetries is generated by the identity and the other by $\tilde{M}$. 
The $N_{c} \rightarrow \infty$ limit is taken in the standard fashion. The Hubbard-Stratonovich fields $\sigma_{i}$ will be chosen to be an arbitrary vector for each node and the same vector for all nodes. Let $V$ be a unitary transformation which diagonalizes the $2 \times 2$ matrix $\tilde{M}$, and let $\sigma \equiv V \tilde{\sigma}$, so that $\left|\sigma_{1}\right|^{2}+\left|\sigma_{2}\right|^{2}=\left|\tilde{\sigma}_{1}\right|^{2}+\left|\tilde{\sigma}_{2}\right|^{2}$. The eigenvalues of $\tilde{M}$ can be rewritten as $m \pm \delta m$, where

$$
m=\frac{g_{0}}{g_{0}^{2}-\vec{g}^{2}} \quad \text { and } \quad \delta m=\frac{\sqrt{\vec{g}^{2}}}{g_{0}^{2}-\vec{g}^{2}}
$$

The modified effective action can be written as

$$
\begin{aligned}
\frac{1}{\beta} S_{\text {eff }}=-N_{c} \int_{-\infty}^{\infty} & \frac{d \omega}{2 \pi} \log \left(-i \omega+\epsilon_{f}+\frac{1}{2} N_{\text {nodes }}\left(\left|\tilde{\sigma}_{1}\right|^{2}+\left|\tilde{\sigma}_{2}\right|^{2}\right) G_{0}(i \omega)\right) \\
& +\frac{1}{2} N_{\text {nodes }} N_{c}\left[\left(\left|\tilde{\sigma}_{1}\right|^{2}+\left|\tilde{\sigma}_{2}\right|^{2}\right) m+\left(\left|\tilde{\sigma}_{1}\right|^{2}-\left|\tilde{\sigma}_{2}\right|^{2}\right) \delta m\right]-Q_{f} \epsilon_{f} \\
=-N_{c} \int_{-\infty}^{\infty} & \frac{d \omega}{2 \pi} \log \left(-i \omega+\epsilon_{f}+\frac{1}{2} N_{\text {nodes }}\left(\left|\tilde{\sigma}_{1}\right|^{2}+\left|\tilde{\sigma}_{2}\right|^{2}\right) G_{0}(i \omega)\right) \\
& +\frac{1}{2} N_{\text {nodes }} N_{c}\left(\frac{\left|\tilde{\sigma}_{1}\right|^{2}}{g_{0}-\sqrt{\vec{g}^{2}}}+\frac{\left|\tilde{\sigma}_{2}\right|^{2}}{g_{0}+\sqrt{\vec{g}^{2}}}\right)-Q_{f} \epsilon_{f}
\end{aligned}
$$

The new Saddle Point Equations are

$$
\frac{Q_{f}}{N_{c}}=-\int_{-\infty}^{\infty} \frac{d \omega}{2 \pi} \frac{1}{-i \omega+\epsilon_{f}+\frac{1}{2} N_{\text {nodes }}\left(\left|\tilde{\sigma}_{1}\right|^{2}+\left|\tilde{\sigma}_{2}\right|^{2}\right) G_{0}(i \omega)}
$$

and

$$
\frac{\tilde{\sigma}_{1}}{g_{0}-\sqrt{\vec{g}^{2}}}=\tilde{\sigma}_{1} \int_{-\infty}^{\infty} \frac{d \omega}{2 \pi} \frac{G_{0}(i \omega)}{-i \omega+\epsilon_{f}+\frac{1}{2} N_{\text {nodes }}\left(\left|\tilde{\sigma}_{1}\right|^{2}+\left|\tilde{\sigma}_{2}\right|^{2}\right) G_{0}(i \omega)}
$$

and

$$
\frac{\tilde{\sigma}_{2}}{g_{0}+\sqrt{\vec{g}^{2}}}=\tilde{\sigma}_{2} \int_{-\infty}^{\infty} \frac{d \omega}{2 \pi} \frac{G_{0}(i \omega)}{-i \omega+\epsilon_{f}+\frac{1}{2} N_{\text {nodes }}\left(\left|\tilde{\sigma}_{1}\right|^{2}+\left|\tilde{\sigma}_{2}\right|^{2}\right) G_{0}(i \omega)}
$$

We now solve these new SPE's. The solution will be cast in the form of a phase diagram which can be plotted in a $\sqrt{\vec{g}^{2}}-g_{0}$ plane (see figure 1). In principle, we find three different solutions of the SPE's:

a ) $\tilde{\sigma}_{1}=\tilde{\sigma}_{2}=0$. This is the region of the phase diagram below the line $g_{0}+\sqrt{\bar{g}^{2}}=g_{c}$. This is the weak-coupling phase. The magnetic impurity is effectively decoupled from the band electrons. To leading order in $1 / N_{c}$ the impurity does not interact with the band fermions and behaves like a free magnetic moment. Consequently the impurity spin suceptibility is infinite, there is no resonance and there is no Kondo screening. 
b ) Both $\tilde{\sigma}_{1} \neq 0$ and $\tilde{\sigma}_{2} \neq 0$. There is no consistent solution of this form unless $\vec{g}^{2}=0$. In other words, this case is possible only if the only non-vanishing coupling constant is $J_{0}$, with $J_{0}>J_{c}$, in which case we do have the $U(2)$ symmetry. This is the line on the axis $g_{0}$ of the figure 1 for $g_{0}>g_{c}$;

c ) $\tilde{\sigma}_{2} \neq 0, \tilde{\sigma}_{1}=0$. A solution of this form satisfies the equations

$$
\begin{aligned}
\frac{1}{g_{0}+\sqrt{\vec{g}^{2}}} & =\int \frac{d \omega}{2 \pi} \frac{G_{0}(i \omega)}{-i \omega+\epsilon_{f}+\frac{1}{2} N_{\text {nodes }}\left|\tilde{\sigma}_{2}\right|^{2} G_{0}(i \omega)} \\
\frac{Q_{f}}{N_{c}} & =-\int \frac{d \omega}{2 \pi} \frac{1}{-i \omega+\epsilon_{f}+\frac{1}{2} N_{\text {nodes }}\left|\tilde{\sigma}_{2}\right|^{2} G_{0}(i \omega)}
\end{aligned}
$$

The action of this solution is given by

$$
\frac{1}{\beta} S_{i m p}=-N_{c} \int \frac{d \omega}{2 \pi} \log \left(-i \omega+\epsilon_{f}+N_{\text {nodes }} \frac{\left|\tilde{\sigma}_{2}\right|^{2}}{2} G_{0}(i \omega)\right)+\frac{N_{c}\left|\tilde{\sigma}_{2}\right|^{2}}{g_{0}+\sqrt{\vec{g}^{2}}}-Q_{f} \epsilon_{f}
$$

For a solution of this type, $\sigma_{1}$ and $\sigma_{2}$ are determined by $\tilde{\sigma}_{1}$ through the relations

$$
\sigma_{1}=\tilde{\sigma}_{2} \frac{\left(g_{1}-i g_{2}\right)}{\sqrt{2 \sqrt{\vec{g}^{2}}\left(\sqrt{\vec{g}^{2}}+g_{3}\right)}} \quad \text { and } \quad \sigma_{2}=-\tilde{\sigma}_{2} \sqrt{\frac{\sqrt{\vec{g}^{2}}+g_{3}}{2 \sqrt{\vec{g}^{2}}}}
$$

This solution is only allowed above the line $g_{0}+\sqrt{\vec{g}^{2}}=g_{c}$ in the phase diagram. This is the Kondo or screening phase. The results of the previous sections apply to this phase.

d ) $\tilde{\sigma}_{2} \neq 0, \tilde{\sigma}_{1}=0$. The SPE's also allow for solutions of this form. In principle such solutions are allowed above the line $g_{0}-\sqrt{\vec{g}^{2}}=g_{c}$. The SPE's for this solution are exactly the same ones found in (c) with $g_{0}+\sqrt{\vec{g}^{2}}$ replaced by $g_{0}-\sqrt{\vec{g}^{2}}$. Clearly this solution competes with the solution of case (c) over a significant region of the phase diagram. In fact its allowed region is completely included within the allowed region for $(c)$. However, we argue that this solution is always metastable and never occurs. In fact, for any finite value of $\sqrt{\vec{g}^{2}}$, the interaction between the impurity and the band electrons will be stronger in case (c) since $g_{0}+\sqrt{\vec{g}^{2}}>g_{0}-\sqrt{\vec{g}^{2}}$, favoring the first case in much the same way in which the bonding state is preferred to the antibonding one 
when the degeneracy in a two-level system is lifted by a perturbation. It is easy to check that this is indeed correct by looking for solutions of both types in the vicinity of the phase transition, for $g_{0}$ close to $g_{c}$ and $\sqrt{\vec{g}^{2}}$ small. In addition, since there is no actual symmetry change between these two "phases", we do not expect a phase transition.

We conclude that the generalized impurity model has just two phases: a phase with an unscreened impurity and a phase with a Kondo effect. The phase boundary is at the line $g_{0}+\sqrt{\vec{g}^{2}}=g_{c}$. The physics of the Kondo phase is almost identical to what was described in the previous sections. In fact, at the level of the $N \rightarrow \infty$ theory, the physical observables of the generalized impurity model can be calculated using the formulas for the single node model of the previous sections. The main difference is that, since the $U(2)$ flavor symmetry at each node is now broken by the explicit form of the impurity Hamiltonian, operators with non-trivial matrix elements in that sector are now allowed. In particular, there will be non-vanishing, finite, off-diagonal matrix elements of the band fermion $T$-matrix. In other words, the impurity will mix band fermion states with different angular momentum. In contrast, processes which mix different nodes are still strictly forbidden. Finally we note that scattering processes of the type described here effectively reduce the number of independent channels. If it were not for the existence of several nodes, we would expect to find a Kondo effect in this phase with a completely screened impurity. Once again, the existence of additional channels associated with the multinode structure turns this into a multichannel Kondo system if inter-node scattering processes are not allowed.

\section{DISCUSSION AND CONCLUSIONS}

In this paper we have considered the problem of flux-phase fermions coupled locally to a single magnetic impurity. This is formally the same as the problem of fermions with a relativistic dispersion in two space dimensions couped to a local spin. We derived explicitely an effective theory in one-space dimension. 
The physics of the effective one-dimensional theory for this problem differs from the conventional radial picture of the Kondo problem in several important ways. Firstly, it is always a multichannel system. The channels reflect the spinor structure of the nodes and the multiplicity of nodes. Secondly, the relativistic dispersion implies a density of states which vanishes linearly at the Fermi energy. Consequently, unlike the conventional Kondo model, the effective one-dimensional problem has a non-local coupling between the effective right movers and the impurity. This is the feature that drives the Kondo effect away from marginality and it is responsible for the phase transition between an unscreened impurity phase and a phase with a Kondo effect. This is consistent with earlier results of Withoff and Fradkin.

However, for the case of the linearly vanishing density of states that we discussed in this paper, the nature of the scaling in the vicinity of this zero-temperature phase transition is drastically changed. We find that all physical quantities exhibit very simple scaling laws modified by logarithmic corrections. We found this behavior in the Kondo scale and in the impurity spin suceptibility. This behavior is strongly reminscent of critical phenomena at an upper critical dimension. One important consequence of the logarithmic corrections is that there are more dynamical scales and that all physical quantities are no longer controlled by the Kondo scale $T_{K}$ alone. This is particularly clear if one compares the amplitude of the $T$-matrix and the position and width of the Kondo resonance.

The model we studied and solved here using large- $N$ methods is an interesting problem on its own right. In a subsequent publication we will report on a study of a similar model for magnetic impurities in $d$-wave superconductors where we will draw heavily on the ideas that we developed here.

It is interesting to compare the effective one-dimensional model that we derived here with the conventional one-dimensional models for the conventional Kondo problem. The standard Kondo problem is equivalent to a model in one space dimension with a single right mover which interacts locally with the impurity spin through the fermion spin density. This coupling through a density is crucial for the physics of the Kondo problem to be 
correctly described by the model of right moving fermions. The fact that the fermions are chiral (namely, only right movers are present) means that, up to a Fermi velocity, the fermion density and current are the same observable. This model can be described entirely in terms of a conserved current. This is the starting point of the Conformal Field Theory approach of Affleck and Ludwig [21]. However, it is also crucial for the success of the approach of Anderson, Yuval and Hamman [3]. In fact, the equivalence that exists between Kondo systems and problems of Macroscopic Quantum Coherence [23] rely heavily on bosonization of models of fermion coupled locally to impurities through a density. The fact that this density is associated to a conserved current means that it cannot acquire anomalous dimensions. Thus, the fixed points of the conventional Kondo contains only marginal operators which are made marginally relevant by quantum fluctuations.

From this analysis it is clear that it is not possible to describe the phase transition that we discuss here in terms of a Conformal Field Theory coupled to local boundary operators representing the impurity. The non-locality of the effective one-dimensional theory is essential. It is because the model is non-local that the operator that couples to the impurity can (and does) acquire an anomalous dimension. This is the mechanism which drives the phase transition. These models are not equivalent to any standard Macroscopic Quantum Tunneling (MQT) model. It may appear that, because the density of states vansihes like a power of the energy, these models could be related to a subohmic MQT system which are known not to have phase transitions. However, subohmic MQT models of quantum impurities coupled to a macroscopic bosonic system, with subohmic spectral density. The models that we discussed here are fermionic and are not equivalent to a subohmic bosonic theory.

\section{ACKNOWLEDGEMENTS}

We are grateful to K. Ingersent for useful discussions and for bringing to our attention the possible physical scenario discussed at the end of Sec. I. This work was supported in part by NSF grants No. NSF DMR94-24511 at the Department of Physics of the University of 
Illinois at Urbana-Champaign, and DMR89-20538/24 at the Materials Research Laboratory of the University of Illinois. 


\section{APPENDIX A:}

In section III and from Eq.(3.8) we have

$$
I_{1}=\frac{1}{\pi} \int_{0}^{e^{-1 / \Delta}} d x \frac{\nu}{\nu^{2}+x^{2}\left(1-\Delta \frac{\log x}{1-x^{2}}\right)^{2}}
$$

and

$$
I_{2}=\frac{1}{\pi} \int_{e^{-1 / \Delta}}^{\infty} d x \frac{\nu}{\nu^{2}+x^{2}\left(1-\Delta \frac{\log x}{1-x^{2}}\right)^{2}}
$$

In the region of integration where $x>>e^{-1 / \Delta}, I_{2}$ can be approximated by

$$
I_{2} \approx \frac{1}{2}-\frac{1}{\pi} \arctan \left(\frac{1}{\nu} e^{-1 / \Delta}\right)
$$

On the other hand, when we are in the regime $x<<e^{-1 / \Delta}$,

$$
I_{1} \approx \frac{\nu}{\pi \Delta^{2}} \int_{0}^{e^{-1 / \Delta}} \frac{d x}{\left(\frac{\nu}{\Delta}\right)^{2}+x^{2} \log ^{2} x} \equiv R(\nu, \Delta)
$$

The following inequality holds

$$
\frac{1}{\pi} \frac{\nu e^{-1 / \Delta}}{\nu^{2}+e^{-2 / \Delta}} \leq R(\nu, \Delta) \leq \frac{1}{\pi \nu} e^{-1 / \Delta}
$$

We will be interested in the following cases.

$$
\begin{aligned}
& \text { If } \quad \nu>>e^{-1 / \Delta}, \quad \text { we have } R(\nu, \Delta) \rightarrow \frac{1}{\pi \nu} e^{-1 / \Delta}<<1 . \\
& \text { If } \quad \nu<<e^{-1 / \Delta}, \quad \text { we have } \quad R(\nu, \Delta) \rightarrow \frac{\nu}{\pi} e^{1 / \Delta}<<1 .
\end{aligned}
$$

For $1>>>>e^{-1 / \Delta}$, we can expand the denominator in Eq.(A4) to get

$$
R(\nu, \Delta) \approx \frac{1}{\pi \nu}\left\{e^{-1 / \Delta}-\frac{3}{\Delta^{2}}\left(\frac{\Delta}{\nu}\right)^{2} e^{-3 / \Delta}+\ldots\right\}
$$

We can now get the behavior of Eq.(3.1) in these two different regimes: for $e^{-1 / \Delta}<<\nu<<1$,

$$
\frac{Q_{f}}{N_{c}} \approx \frac{1}{2}-\frac{3}{\pi \nu^{3}} e^{-3 / \Delta} \text { and } \nu \approx\left(\frac{3}{\pi}\right)^{1 / 3} \frac{e^{-1 / \Delta}}{\left(-\frac{Q_{f}}{N_{c}}+\frac{1}{2}\right)^{1 / 3}}>>e^{-1 / \Delta} \text { if } \frac{Q_{f}}{N_{c}} \rightarrow \frac{1}{2}
$$


the other limit corresponds to $\nu<<e^{-1 / \Delta}<<1$; consequently the contribution from $I_{2}$ is neglegible and

$$
\frac{Q_{f}}{N_{c}} \approx \frac{\nu}{\pi} e^{1 / \Delta} \text { or } \quad \nu \approx \pi \frac{Q_{f}}{N_{c}} e^{-1 / \Delta}<<e^{-1 / \Delta} \quad \text { if } \quad \frac{Q_{f}}{N_{c}}<<\frac{1}{\pi}
$$

Now we want to work out similar approximations for the other SPE. We have

$$
\begin{aligned}
I_{1}^{\prime} & \equiv-\int_{0}^{e^{-1 / \Delta}} d x \frac{\log x}{1-x^{2}}\left(1-\Delta \frac{\log x}{1-x^{2}}\right) \frac{x^{2}}{\nu^{2}+x^{2}\left(1-\Delta \frac{\log x}{1-x^{2}}\right)^{2}} \\
& \approx \frac{1}{\Delta} e^{-1 / \Delta}-\frac{\pi \nu}{\Delta} R(\nu, \Delta)
\end{aligned}
$$

in which we made use of the fact that over the integration interval, the variable $x$ is very small, so that the approximations $1-x^{2} \approx 1$ and $-\Delta \log x>>1$ are consistent. For the other portion of the integral (i.e., for $I_{2}^{\prime}$ ) we are in a situation where $x>e^{-1 / \Delta}$ and then $-\Delta \frac{\log x}{1-x^{2}}<1$, so we neglect the logaritmic term against 1 .

We need here the following result

$$
\int_{0}^{\infty} d x \frac{x^{2} \log x}{1-x^{2}} \frac{1}{\nu^{2}+x^{2}}=\frac{1}{2} \frac{1}{1+\nu^{2}}\left\{\frac{\pi^{2}}{2}+\pi \nu \log \nu\right\}
$$

Then

$$
\begin{aligned}
& I_{2}^{\prime} \equiv-\int_{e^{-1 / \Delta}}^{\infty} d x \frac{\log x}{1-x^{2}}\left(1-\Delta \frac{\log x}{1-x^{2}}\right) \frac{x^{2}}{\nu^{2}+x^{2}\left(1-\Delta \frac{\log x}{1-x^{2}}\right)^{2}} \\
& \approx-\int_{e^{-1 / \Delta}}^{\infty} d x \frac{x^{2} \log x}{1-x^{2}} \frac{1}{\nu^{2}+x^{2}} \\
& \approx \frac{1}{2} \frac{1}{1+\nu^{2}}\left\{\frac{\pi^{2}}{2}+\pi \nu \log \nu\right\}-e^{-1 / \Delta}\left(1+\frac{1}{\Delta}\right) \\
& \quad-\nu \log \nu \arctan \left(\frac{1}{\nu} e^{-1 / \Delta}\right)-\nu \mathrm{F}\left(\frac{1}{\nu} e^{-1 / \Delta}\right)
\end{aligned}
$$

The function $\mathrm{F}(z)$ verifies that, for $|z|>1, \mathrm{~F}(z)=\mathrm{F}\left(\frac{1}{z}\right)$ and for $|z|<1$ is given by

$$
\begin{aligned}
\mathrm{F}(z)=\sum_{n=0}^{\infty}(-1)^{n} & \frac{1}{2 n+1} z^{2 n+1}\left[\log z-\frac{1}{2 n+1}\right] \\
& \approx z(\log z-1)-\frac{1}{3} z^{3}\left(\log z-\frac{1}{3}\right)+\ldots
\end{aligned}
$$

Getting everything together, in the limit $\nu \gg e^{-1 / \Delta}$ and $\nu, \Delta<<1$, where 


$$
R(\nu, \Delta) \rightarrow \frac{1}{\pi \nu} e^{-1 / \Delta}
$$

one can write the SPE in the form

$$
\frac{1}{g_{0}} \approx \frac{\pi^{2}}{4}+\frac{\pi}{2} \nu \log \nu
$$

which can be solved by iteration (see Eq.(3.14) in section [II). The other limiting case corresponds to $\nu e^{1 / \Delta}<<1$, with $\nu, \Delta<<1$. Here

$$
R(\nu, \Delta) \rightarrow \frac{\nu}{\pi} e^{1 / \Delta}
$$

The SPE now is

$$
\frac{\pi^{2}}{4}-\frac{1}{g_{0}} \approx e^{-1 / \Delta}\left[1+\frac{1}{\Delta}\left(\pi \frac{Q_{f}}{N_{c}}\right)^{2}+\left(\pi \frac{Q_{f}}{N_{c}}\right)^{3}+\left(\pi \frac{Q_{f}}{N_{c}}\right)^{2} \log \left(\pi \frac{Q_{f}}{N_{c}}\right)\right]
$$

since in this case, $\nu e^{1 / \Delta} \approx \pi \frac{Q_{f}}{N_{c}}$. The cubic term can be dropped rightaway. As for the other terms, as we approach the transition, $\pi \frac{Q_{f}}{N_{c}}$ remains fixed while $\Delta \rightarrow 0$. Therefore, the leading term is going to be the one with the factor $\frac{1}{\Delta}$. This immediately gives the implicit expression for $\Delta$ in terms of the impurity occupancy $\frac{Q_{f}}{N_{c}}$ and the distance to the critical point $\frac{1}{g_{c}}-\frac{1}{g_{0}}$ that we used in Eq.(3.9) in section [II. 


\section{REFERENCES}

[1] J. Kondo, Prog. Theor. Phys. 32, 37 (1964).

[2] P. W. Anderson, J. Physc. C 3, 2436 (1970).

[3] P. W. Anderson, G. Yuval, and D. R. Hamann, Phys. Rev. B1, 4464 (1970).

[4] K. G. Wilson, Rev. Mod. Phys. 47, 773 (1975).

[5] N. Andrei, Phys. Rev. Lett. 45, 379 (1980).

[6] P. B. Wiegmann, JETP Lett. 31, 367 (1980).

[7] N. Read and D. M. Newns, J. Phys. C 16, 3273 (1983).

[8] P. Coleman, Phys. Rev. B29, 3035 (1984); J. Mag. Mat. 47, 323 (1985).

[9] D. Withoff and E. Fradkin, Phys. Rev. Lett. 64, 1835 (1990); D. J. Withoff, Path Integral Methods for the large-N Kondo Model, University of Illinois Thesis, 1988.

[10] I. K. Affleck and J. B. Marston, Phys. Rev. B37, 3774 (1988).

[11] See for example the review by D. J. Scalapino, Phys. Rep. 250, 329 (1995).

[12] J. R. Schrieffer, Theory of Superconductivity, Addison-Wesley, Redwood City (1983).

[13] A. A. Nersesyan, A. M. Tsvelik and F. Wenger, Phys. Rev. Lett. 72, 2628 (1994); Nucl. Phys. B438, 561 (1995).

[14] L. S. Borkowski and P. J. Hirschfeld, Phys. Rev. B46, 9274 (1992).

[15] C. Cassanello and E. Fradkin, in preparation.

[16] The usual logarithmic divergencies of the high temperature expansion of the standard Kondo problem are absent for this non-marginal system. The logarithmic corrections that we discuss have a completely different and new character.

[17] N. Andrei and C. Destri, Phys. Rev. Lett. 52, 364 (1984); P. B. Wiegmann and 
A. M. Tsvelik, Z. Phys. B54, 201 (1985) .

[18] P. Nozières and A. Blandin, J. Physique 41, 193 (1980).

[19] After this paper was submitted for publication, K. Ingersent ( K. Ingersent, private communication) has kindly pointed out to us that in a recent work of his he has investigated similar issues using a Wilson RG approach. Ingersent's RG suggests that our phase transition may actually be suppressed.

[20] S. Kim, A. Kocić and J. Kogut, Nucl.Phys. B429, 407 (1994); A. Kocić and J. Kogut, University of Illinois preprint, 1995.

[21] I. A. Affleck and A. W. W. Ludwig, Nucl. Phys. B352, 849 (1991).

[22] D. Withoff and E. Fradkin, Phys. Rev. B34, 8172 (1986).

[23] For a review, see A. J. Leggett et al, Rev. Mod. Phys. 59, 1 (1987). 


\section{FIGURES}

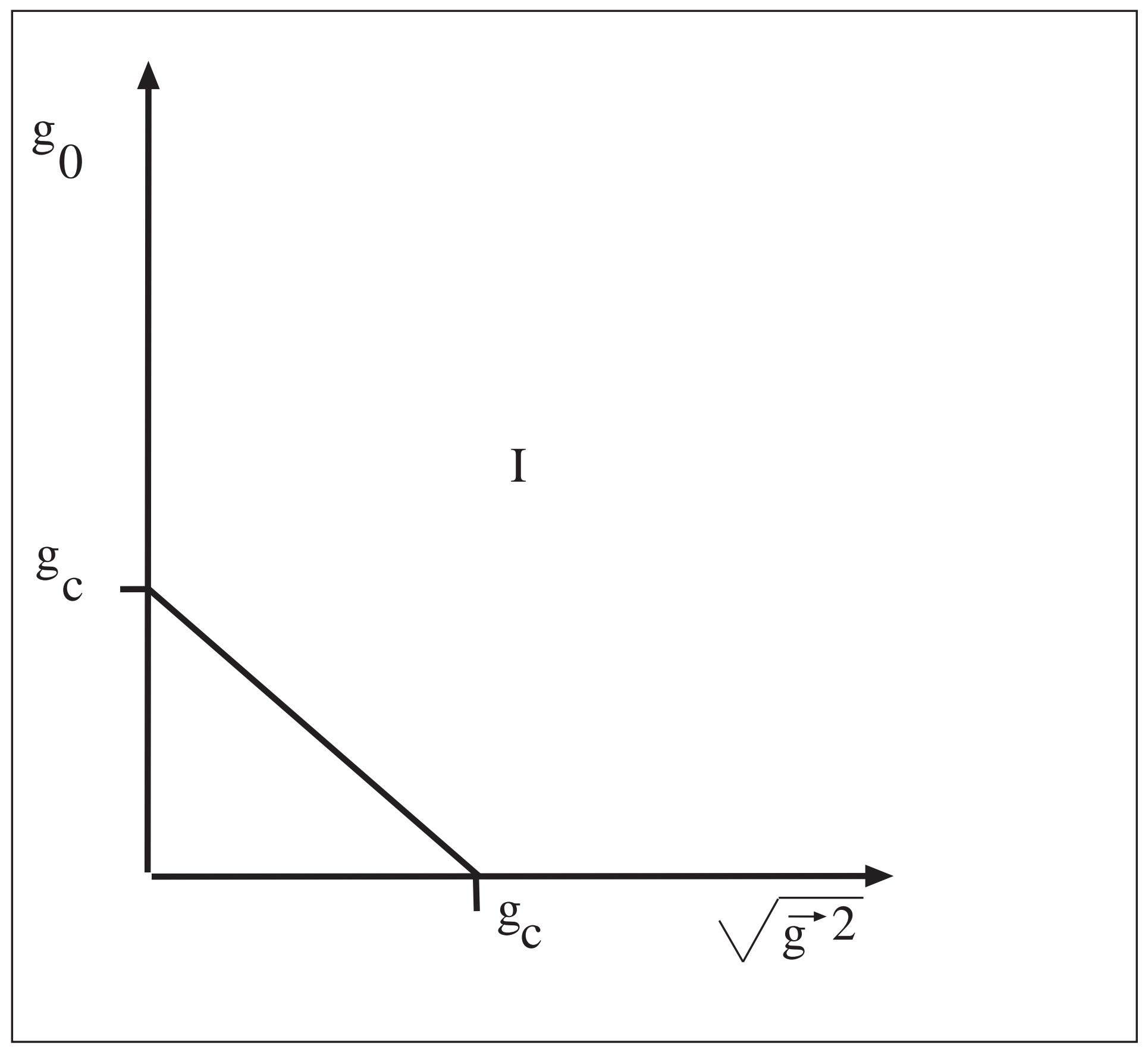

FIG. 1. Phase diagram of the generalized impurity model. 